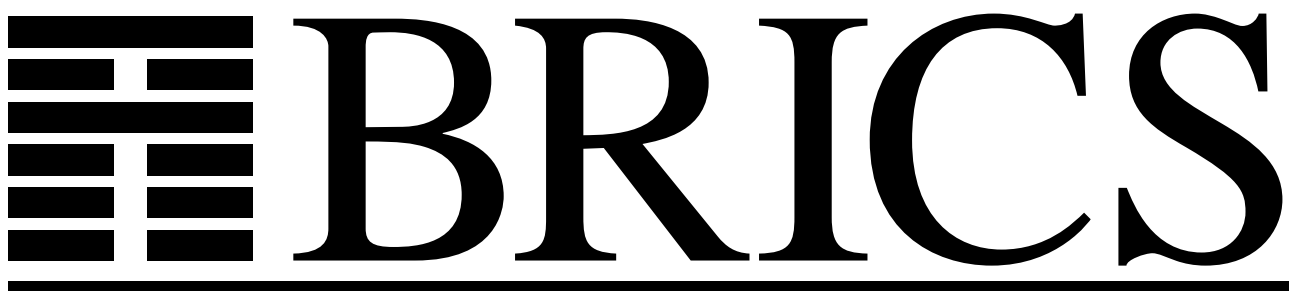

Basic Research in Computer Science

\title{
An Operational Foundation for Delimited Continuations in the CPS Hierarchy
}

Małgorzata Biernacka

Dariusz Biernacki

Olivier Danvy

RS-05-11

ISSN 0909-0878

March 2005 
Copyright (c) 2005, Małgorzata Biernacka \& Dariusz Biernacki \& Olivier Danvy.

BRICS, Department of Computer Science University of Aarhus. All rights reserved.

Reproduction of all or part of this work is permitted for educational or research use on condition that this copyright notice is included in any copy.

See back inner page for a list of recent BRICS Report Series publications. Copies may be obtained by contacting:

\author{
BRICS \\ Department of Computer Science \\ University of Aarhus \\ Ny Munkegade, building 540 \\ DK-8000 Aarhus C \\ Denmark \\ Telephone: +45 89423360 \\ Telefax: $\quad+4589423255$ \\ Internet: BRICS@brics.dk
}

BRICS publications are in general accessible through the World Wide Web and anonymous FTP through these URLs:

http://www.brics.dk

ftp://ftp.brics.dk

This document in subdirectory RS / 05/11/ 


\title{
An Operational Foundation for Delimited Continuations in the CPS Hierarchy *
}

\author{
Małgorzata Biernacka, Dariusz Biernacki, and Olivier Danvy \\ $\mathrm{BRICS}^{\dagger}$ \\ Department of Computer Science \\ University of Aarhus $\ddagger$
}

March 21, 2005

\begin{abstract}
We present an abstract machine and a reduction semantics for the $\lambda$-calculus extended with control operators that give access to delimited continuations in the CPS hierarchy. The abstract machine is derived from an evaluator in continuation-passing style (CPS); the reduction semantics (i.e., a small-step operational semantics with an explicit representation of evaluation contexts) is constructed from the abstract machine; and the control operators are the shift and reset family. At level $n$ of the CPS hierarchy, programs can use the control operators $\operatorname{shift}_{i}$ and $\operatorname{reset}_{i}$ for $1 \leq i \leq n$, the evaluator has $n+1$ layers of continuations, the abstract machine has $n+1$ layers of control stacks, and the reduction semantics has $n+1$ layers of evaluation contexts.

We also present new applications of delimited continuations in the CPS hierarchy: finding list prefixes and normalization by evaluation for a hierarchical language of units and products.
\end{abstract}

\footnotetext{
* A preliminary version was presented at the Fourth ACM SIGPLAN Workshop on Continuations [15].

$\dagger$ Basic Research in Computer Science (www.brics.dk), funded by the Danish National Research Foundation.

$\ddagger$ IT-parken, Aabogade 34, DK-8200 Aarhus N, Denmark.

E-mail: \{mbiernac, dabi, danvy\}@brics.dk

Home pages: http://www.brics.dk/ $\{$ mbiernac, dabi, danvy $\}$
} 


\section{Contents}

1 Introduction 1

2 From evaluator to reduction semantics for arithmetic expressions 1

2.1 The starting point: an evaluator in direct style . . . . . . . . . . . . . 2

2.2 CPS transformation . . . . . . . . . . . . . . . . . 2

2.3 Defunctionalization . . . . . . . . . . . . . . . . . . 2

2.4 Abstract machines as defunctionalized continuation-passing programs . . . . 4

2.5 From value-based abstract machine to term-based abstract machine . . . . . 4

2.6 From term-based abstract machine to reduction semantics . . . . . . . . . . 4

2.7 From reduction semantics to term-based abstract machine . . . . . . . . . 6

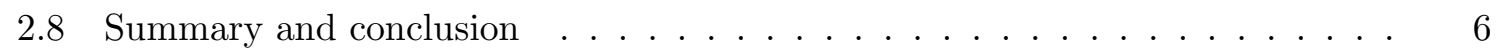

3 Programming with delimited continuations $\quad 6$

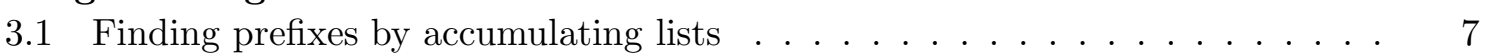

3.2 Finding prefixes by accumulating list constructors $\ldots \ldots \ldots \ldots$

3.3 Finding prefixes in direct style . . . . . . . . . . . . . . 8

3.4 Finding prefixes in continuation-passing style . . . . . . . . . . . 9

3.5 The CPS hierarchy . . . . . . . . . . . . . . . . . . . 10

3.6 A note about typing . . . . . . . . . . . . . . . . 10

3.7 Related work . . . . . . . . . . . . . . . . . . . . 10

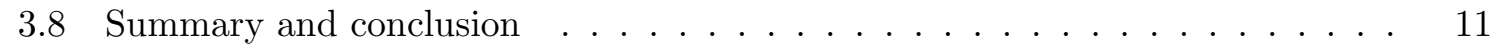

4 From evaluator to reduction semantics for delimited continuations 11

4.1 An environment-based evaluator . . . . . . . . . . . . . . 11

4.2 An environment-based abstract machine . . . . . . . . . . . . . . . 13

4.3 A substitution-based abstract machine . . . . . . . . . . . . . . . 15

4.4 A reduction semantics . . . . . . . . . . . . . . . . 17

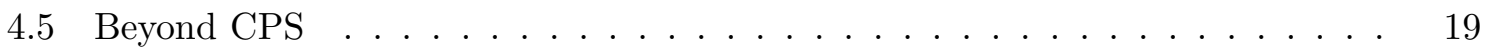

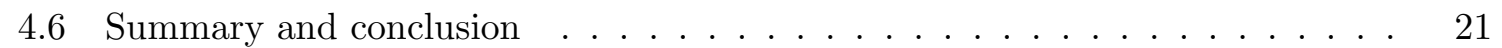

5 From evaluator to reduction semantics for the CPS hierarchy 21

5.1 An environment-based evaluator . . . . . . . . . . . . . 21

5.2 An environment-based abstract machine . . . . . . . . . . . . 23

5.3 A substitution-based abstract machine . . . . . . . . . . . . 25

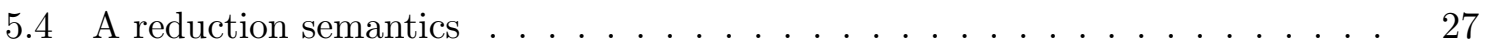

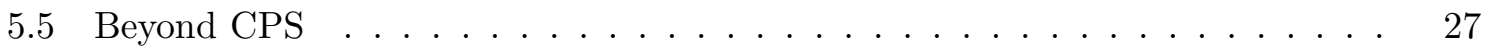

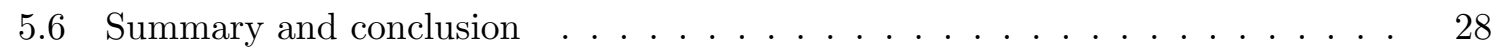

6 Programming in the CPS hierarchy 28

6.1 Normalization by evaluation . . . . . . . . . . . . . . . 28

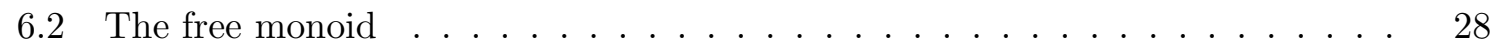

6.3 A language of propositions . . . . . . . . . . . . . . . . . 29

6.4 A hierarchical language of units and products . . . . . . . . . . . . . 31

6.5 A note about efficiency . . . . . . . . . . . . . . . . . . . . . . 34

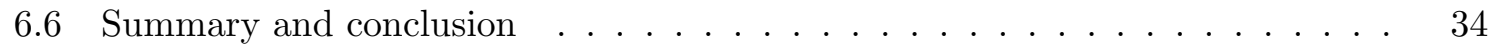




\section{List of Figures}

1 A direct-style evaluator for arithmetic expressions . . . . . . . . . . . 3

2 A continuation-passing evaluator for arithmetic expressions . . . . . . . 3

3 A defunctionalized continuation-passing evaluator for arithmetic expressions . 3

4 A value-based abstract machine for evaluating arithmetic expressions . . . . . 5

5 A term-based abstract machine for processing arithmetic expressions . . . . . 5

6 An environment-based evaluator for the first level of the CPS hierarchy . . . 12

7 An environment-based abstract machine for the first level of the CPS hierarchy 14

8 A substitution-based abstract machine for the first level of the CPS hierarchy 15

$9 \quad$ An environment-based evaluator for the CPS hierarchy at level $n \ldots \ldots$. . . 21

10 An environment-based evaluator for the CPS hierarchy at level $n$, ctd. . . . . 22

11 An environment-based abstract machine for the CPS hierarchy at level $n$. . 23

12 An environment-based abstract machine for the CPS hierarchy at level $n$, ctd. 24

13 A substitution-based abstract machine for the CPS hierarchy at level $n \ldots 25$

14 A substitution-based abstract machine for the the CPS hierarchy at level $n$, ctd. 26 


\section{Introduction}

The studies of delimited continuations can be classified in two groups: those that use continuation-passing style (CPS) and those that rely on operational intuitions about control instead. Of the latter, there is a large number proposing a variety of control operators $[5,36,39,40$, $47,50,51,61,66,70,77]$ which have found applications in models of control, concurrency, and type-directed partial evaluation $[8,50,71]$. Of the former, there is the work revolving around the family of control operators shift and reset [26-28, 31, 41, 42, 53, 54,62, 77] which have found applications in non-deterministic programming, code generation, partial evaluation, normalization by evaluation, computational monads, and mobile computing $[6,7,9,16,21,22$, $32,33,43,44,46,49,55,58,68,73,74,76]$.

The original motivation for shift and reset was a continuation-based programming pattern involving several layers of continuations. The original specification of these operators relied both on a repeated CPS transformation and on an evaluator with several layers of continuations (as is obtained by repeatedly transforming a direct-style evaluator into continuationpassing style). Only subsequently have shift and reset been specified operationally, by developing operational analogues of a continuation semantics and of the CPS transformation [31].

The goal of our work here is to establish a new operational foundation for delimited continuations, using CPS as a guideline. To this end, we start with the original evaluator for shift $_{1}$ and reset $_{1}$. This evaluator uses two layers of continuations: a continuation and a meta-continuation. We then defunctionalize it into an abstract machine [1] and we construct the corresponding reduction semantics [35], as pioneered by Felleisen and Friedman [38]. The development scales to shift ${ }_{n}$ and reset $_{n}$. It is reusable for any control operators that are compatible with CPS, i.e., that can be characterized with a (possibly iterated) CPS translation or with a continuation-based evaluator. It also pinpoints where operational intuitions go beyond CPS.

This article is structured as follows. In Section 2, we review the enabling technology of our work: Reynolds's defunctionalization, the observation that a defunctionalized CPS program implements an abstract machine, and the observation that Felleisen's evaluation contexts are the defunctionalized continuations of a continuation-passing evaluator; we demonstrate this enabling technology on a simple example, arithmetic expressions. In Section 3, we illustrate the use of shift and reset with the classic example of finding list prefixes, using an MLlike programming language. In Section 4, we then present our main result: starting from the original evaluator for shift and reset, we defunctionalize it into an abstract machine; we analyze this abstract machine and construct the corresponding reduction semantics. In Section 5, we extend this result to the CPS hierarchy. In Section 6, we illustrate the CPS hierarchy with a class of normalization functions for a hierarchical language of units and products.

\section{From evaluator to reduction semantics for arithmetic expressions}

We demonstrate the derivation from an evaluator to a reduction semantics. The derivation consists of the following steps:

1. we start from an evaluator for a given language; if it is in direct style, we CPS-transform it; 
2. we defunctionalize the CPS evaluator, obtaining a value-based abstract machine;

3. we modify the abstract machine to make it term-based instead of value-based; in particular, if the evaluator uses an environment, then so does the corresponding value-based abstract machine, and in that case, making the machine term-based leads us to use substitutions rather than an environment;

4. we analyze the transitions of the term-based abstract machine to identify the evaluation strategy it implements and the set of reductions it performs; the result is a reduction semantics.

The first two steps are based on previous work on a functional correspondence between evaluators and abstract machines [1-3,16,25], which itself is based on Reynolds's seminal work on definitional interpreters [67]. The last two steps follow the lines of Felleisen and Friedman's original work on a reduction semantics for the call-by-value $\lambda$-calculus extended with control operators [38]. The last step has been studied further by Hardin, Maranget, and Pagano [48] in the context of explicit substitutions and by Danvy and Nielsen [30].

In the rest of this section, our running example is the language of arithmetic expressions, formed using natural numbers (the values) and additions (the computations):

$$
\exp \ni e::=\ulcorner m\urcorner \mid e_{1}+e_{2}
$$

\subsection{The starting point: an evaluator in direct style}

We define an evaluation function for arithmetic expressions by structural induction on their syntax. The resulting direct-style evaluator is displayed in Figure 1.

\subsection{CPS transformation}

We CPS-transform the evaluator by naming intermediate results, sequentializing their computation, and introducing an extra functional parameter, the continuation $[28,64,72]$. The resulting continuation-passing evaluator is displayed in Figure 2.

\subsection{Defunctionalization}

The generalization of closure conversion [57] to defunctionalization is due to Reynolds [67]. The goal is to represent a functional value with a first-order data structure. The means is to partition the function space into a first-order sum where each summand corresponds to a lambda-abstraction in the program. In a defunctionalized program, function introduction is thus represented as an injection, and function elimination as a call to a first-order apply function implementing a case dispatch. In an ML-like functional language, sums are represented as data types, injections as data-type constructors, and apply functions are defined by case over the corresponding data types [29].

Here, we defunctionalize the continuation of the continuation-passing evaluator in Figure 2. We thus need to define a first-order algebraic data type and its apply function. To this end, we enumerate the lambda-abstractions that give rise to the inhabitants of this function space; there are three: the initial continuation in evaluate and the two continuations in eval. The initial continuation is closed, and therefore the corresponding algebraic constructor is nullary. The two other continuations have two free variables, and therefore the corresponding 
- Values: $\quad$ val $\ni v::=m$

- Evaluation function: eval : exp $\rightarrow$ val

$$
\begin{aligned}
\text { eval }(\ulcorner m\urcorner) & =m \\
\text { eval }\left(e_{1}+e_{2}\right) & =\text { eval }\left(e_{1}\right)+\text { eval }\left(e_{2}\right)
\end{aligned}
$$

- Main function: evaluate : $\exp \rightarrow$ val

$$
\text { evaluate }(e)=\text { eval }(e)
$$

Figure 1: A direct-style evaluator for arithmetic expressions

- Values: val $\ni v::=m$

- Continuations: cont $=$ val $\rightarrow$ val

- Evaluation function: eval : $\exp \times$ cont $\rightarrow$ val

$$
\begin{aligned}
\text { eval }(\ulcorner m\urcorner, k) & =k m \\
\operatorname{eval}\left(e_{1}+e_{2}, k\right) & =\operatorname{eval}\left(e_{1}, \lambda m_{1} \text {. eval }\left(e_{2}, \lambda m_{2} \cdot k\left(m_{1}+m_{2}\right)\right)\right)
\end{aligned}
$$

- Main function: evaluate : exp $\rightarrow$ val

$$
\text { evaluate }(e)=\operatorname{eval}(e, \lambda v \cdot v)
$$

Figure 2: A continuation-passing evaluator for arithmetic expressions

- Values: val $\ni v::=m$

- Defunctionalized continuations: cont $\ni k::=[]\left|\operatorname{ADD}_{2}(e, k)\right| \operatorname{ADD}_{1}(v, k)$

- Functions eval : exp $\times$ cont $\rightarrow$ val and apply_cont $:$ cont $\times$ val $\rightarrow$ val:

$$
\begin{aligned}
\text { eval }(\ulcorner m\urcorner, k) & =\text { apply_cont }(k, m) \\
\text { eval }\left(e_{1}+e_{2}, k\right) & =\text { eval }\left(e_{1}, \operatorname{ADD}_{2}\left(e_{2}, k\right)\right) \\
\text { apply_cont }([], v) & =v \\
\text { apply_cont }\left(\mathrm{ADD}_{2}\left(e_{2}, k\right), v_{1}\right) & =\text { eval }\left(e_{2}, \operatorname{ADD}_{1}\left(v_{1}, k\right)\right) \\
\text { apply_cont }\left(\mathrm{ADD}_{1}\left(m_{1}, k\right), m_{2}\right) & =\text { apply_cont }\left(k, m_{1}+m_{2}\right)
\end{aligned}
$$

- Main function: evaluate : $\exp \rightarrow$ val

$$
\text { evaluate }(e)=\operatorname{eval}(e,[])
$$

Figure 3: A defunctionalized continuation-passing evaluator for arithmetic expressions 
constructors are binary. As for the apply function, it interprets the algebraic constructors. The resulting defunctionalized evaluator is displayed in Figure 3.

\subsection{Abstract machines as defunctionalized continuation-passing programs}

Elsewhere $[1,25]$, we have observed that a defunctionalized continuation-passing program implements an abstract machine: each configuration is the name of a function together with its arguments, and each function clause represents a transition. (As a corollary, we have also observed that the defunctionalized continuation of an evaluator forms what is known as an 'evaluation context' $[24,29,38]$.)

Indeed Plotkin's Indifference Theorem [64] states that continuation-passing programs are independent of their evaluation order. In Reynolds's words [67], all the subterms in applications are 'trivial'; and in Moggi's words [60], these subterms are values and not computations. Furthermore, continuation-passing programs are tail recursive [72]. Therefore, since in a continuation-passing program all calls are tail calls and all subcomputations are elementary, a defunctionalized continuation-passing program implements a transition system [65], i.e., an abstract machine.

We thus reformat Figure 3 into Figure 4 . The correctness of the abstract machine with respect to the initial evaluator follows from the correctness of CPS transformation and of defunctionalization.

\subsection{From value-based abstract machine to term-based abstract machine}

We observe that the domain of expressible values in Figure 4 can be embedded in the syntactic domain of expressions. We therefore adapt the abstract machine to work on terms rather than on values. The result is displayed in Figure 5; it is a syntactic theory [35].

\subsection{From term-based abstract machine to reduction semantics}

The method of deriving a reduction semantics from an abstract machine was introduced by Felleisen and Friedman [38] to give a reduction semantics for control operators. Let us demonstrate it.

We analyze the transitions of the abstract machine in Figure 5. The second component of eval-transitions - the stack representing "the rest of the computation" - has already been identified as the evaluation context of the currently processed expression. We thus read a configuration $\langle e, C\rangle_{\text {eval }}$ as a decomposition of some expression into a sub-expression $e$ and an evaluation context $C$.

Next, we identify the reduction and decomposition rules in the transitions of the machine. Since a configuration can be read as a decomposition, we compare the left-hand side and the right-hand side of each transition. If they represent the same expression, then the given transition defines a decomposition (i.e., it searches for the next redex according to some evaluation strategy); otherwise we have found a redex. Moreover, reading the decomposition rules from right to left defines a 'plug' function that reconstructs an expression from its decomposition.

Here the decomposition function as read off the abstract machine is total. In general, however, it may be undefined for stuck terms; one can then extend it straightforwardly into a total function that decomposes a term into a context and a potential redex, i.e., an actual redex (as read off the machine), or a stuck redex. 
- Values: $\quad v::=m$

- Evaluation contexts: $C::=[]\left|\mathrm{ADD}_{2}(e, C)\right| \mathrm{ADD}_{1}(v, C)$

- Initial transition, transition rules, and final transition:

\begin{aligned} &$e \Rightarrow\langle e,[]\rangle_{\text {eval }} \\ &$\hline$\langle\ulcorner m\urcorner, C\rangle_{\text {eval }} \Rightarrow\langle C, m\rangle_{\text {cont }} \\ &\left\langle e_{1}+e_{2}, C\right\rangle_{\text {eval }} \Rightarrow\left\langle e_{1}, \mathrm{ADD}_{2}\left(e_{2}, C\right)\right\rangle_{\text {eval }} \\ &$\hline$\left\langle\mathrm{ADD}_{2}\left(e_{2}, C\right), v_{1}\right\rangle_{\text {cont }} \Rightarrow\left\langle e_{2}, \mathrm{ADD}_{1}\left(v_{1}, C\right)\right\rangle_{\text {eval }} \\ &\left\langle\mathrm{ADD}_{1}\left(m_{1}, C\right), m_{2}\right\rangle_{\text {cont }} \Rightarrow\left\langle C, m_{1}+m_{2}\right\rangle_{\text {cont }} \\ &$\hline$\langle[], v\rangle_{\text {cont }} \Rightarrow v \\ &$\hline\end{aligned}

Figure 4: A value-based abstract machine for evaluating arithmetic expressions

- Expressions and values: $e::=v \mid e_{1}+e_{2}$

$$
v::=\ulcorner m\urcorner
$$

- Evaluation contexts: $C::=[]\left|\mathrm{ADD}_{2}(e, C)\right| \mathrm{ADD}_{1}(v, C)$

- Initial transition, transition rules, and final transition:

\begin{aligned} &$e \Rightarrow\langle e,[]\rangle_{\text {eval }} \\ &$\hline$\langle\ulcorner m\urcorner, C\rangle_{\text {eval }} \Rightarrow\langle C,\ulcorner m\urcorner\rangle_{\text {cont }} \\ &\left\langle e_{1}+e_{2}, C\right\rangle_{\text {eval }} \Rightarrow\left\langle e_{1}, \operatorname{ADD}_{2}\left(e_{2}, C\right)\right\rangle_{\text {eval }} \\ &$\hline$\left\langle\mathrm{ADD}_{2}\left(e_{2}, C\right), v_{1}\right\rangle_{\text {cont }} \Rightarrow\left\langle e_{2}, \mathrm{ADD}_{1}\left(v_{1}, C\right)\right\rangle_{\text {eval }} \\ &\left\langle\mathrm{ADD}_{1}\left(\left\ulcorner m_{1}\right\urcorner, C\right),\left\ulcorner m_{2}\right\urcorner\right\rangle_{\text {cont }} \Rightarrow\left\langle C,\left\ulcorner m_{1}+m_{2}\right\urcorner\right\rangle_{\text {cont }} \\ &$\hline$\langle[], v\rangle_{\text {cont }} \Rightarrow v \\ &$\hline\end{aligned}

Figure 5: A term-based abstract machine for processing arithmetic expressions 
In this simple example there is only one reduction rule. This rule performs the addition of natural numbers:

$$
\text { (add) } C\left[\left\ulcorner m_{1}\right\urcorner+\left\ulcorner m_{2}\right\urcorner\right] \rightarrow C\left[\left\ulcorner m_{1}+m_{2}\right\urcorner\right]
$$

The remaining transitions decompose an expression according to the left-to-right strategy.

\subsection{From reduction semantics to term-based abstract machine}

In Section 2.6, we have constructed the reduction semantics corresponding to the abstract machine of Figure 5, as pioneered by Felleisen and Friedman [37,38]. Over the last few years $[23,30]$, Danvy and Nielsen have studied the converse transformation and systematized the construction of an abstract machine from a reduction semantics. The main idea is to short-cut the decompose-contract-plug loop, in the definition of evaluation as the transitive closure of one-step reduction, into a refocus-contract loop. The refocus function is constructed as an efficient (i.e., deforested) composition of plug and decompose that maps a term and a context either to a value or to a redex and a context. The result is a 'pre-abstract machine' computing the transitive closure of the refocus function. This pre-abstract machine can then be simplified into an eval/apply abstract machine.

It is simple to verify that using refocusing, one can go from the reduction semantics of Section 2.6 to the eval/apply abstract machine of Figure 5.

\subsection{Summary and conclusion}

We have demonstrated how to derive an abstract machine out of an evaluator, and how to construct the corresponding reduction semantics out of this abstract machine. In Section 4, we apply this derivation and this construction to the first level of the CPS hierarchy, and in Section 5, we apply them to an arbitrary level of the CPS hierarchy. But first, let us illustrate how to program with delimited continuations.

\section{Programming with delimited continuations}

We present two examples of programming with delimited continuations. Given a list $x s$ and a predicate $p$, we want

1. to find the first prefix of $x s$ whose last element satisfies $p$, and

2. to find all such prefixes of $x s$.

For example, given the predicate $\lambda m . m>2$ and the list $[0,3,1,4,2,5]$, the first prefix is $[0,3]$ and the list of all the prefixes is $[[0,3],[0,3,1,4],[0,3,1,4,2,5]]$.

In Section 3.1, we start with a simple solution that uses a first-order accumulator. This simple solution is in defunctionalized form. In Section 3.2, we present its higher-order counterpart, which uses a functional accumulator. This functional accumulator acts as a delimited continuation. In Section 3.3, we present its direct-style counterpart (which uses shift and reset) and in Section 3.4, we present its continuation-passing counterpart (which uses two layers of continuations). In Section 3.5, we introduce the CPS hierarchy informally. We then mention a typing issue in Section 3.6 and review related work in Section 3.7. 


\subsection{Finding prefixes by accumulating lists}

A simple solution is to accumulate the prefix of the given list in reverse order while traversing this list and testing each of its elements:

- if no element satisfies the predicate, there is no prefix and the result is the empty list;

- otherwise, the prefix is the reverse of the accumulator.

$$
\begin{aligned}
& \text { find_first_prefix_a }(p, x s) \stackrel{\text { def }}{=} \text { letrec visit }(n i l, a) \\
& =\text { nil } \\
& \text { | visit }(x:: x s, a) \\
& =\text { let } a^{\prime}=x:: a \\
& \text { in if } p x \\
& \text { then reverse }\left(a^{\prime}, \text { nil }\right) \\
& \text { else visit }\left(x s, a^{\prime}\right) \\
& \text { and reverse (nil, xs) } \\
& =x s \\
& \text { | reverse }(x:: a, x s) \\
& =\operatorname{reverse}(a, x:: x s) \\
& \text { in visit (xs, nil) } \\
& \text { find_all_prefixes_a }(p, x s) \stackrel{\text { def }}{=} \text { letrec visit }(n i l, a) \\
& =\text { nil } \\
& \mid \text { visit }(x:: x s, a) \\
& =\text { let } a^{\prime}=x:: a \\
& \text { in if } p x \\
& \text { then }\left(\text { reverse }\left(a^{\prime}, \text { nil }\right)\right)::\left(\text { visit }\left(x s, a^{\prime}\right)\right) \\
& \text { else visit }\left(x s, a^{\prime}\right) \\
& \text { and reverse (nil, xs) } \\
& =x s \\
& \text { | reverse }(x:: a, x s) \\
& =\text { reverse }(a, x:: x s) \\
& \text { in visit }(x s, \text { nil) }
\end{aligned}
$$

To find the first prefix, one stops as soon as a satisfactory list element is found. To list all the prefixes, one continues the traversal, adding the current prefix to the list of the remaining prefixes.

We observe that the two solutions are in defunctionalized form [29,67]: the accumulator has the data type of a defunctionalized function and reverse is its apply function. We present its higher-order counterpart next [52].

\subsection{Finding prefixes by accumulating list constructors}

Instead of accumulating the prefix in reverse order while traversing the given list, we accumulate a function constructing the prefix:

- if no element satisfies the predicate, the result is the empty list; 
- otherwise, we apply the functional accumulator to construct the prefix.

$$
\begin{aligned}
& \text { find_first_prefix_c } c_{1}(p, x s) \stackrel{\text { def }}{=} \text { letrec visit }(n i l, k) \\
& =\text { nil } \\
& \mid \operatorname{visit}(x:: x s, k) \\
& =\text { let } k^{\prime}=\lambda v s . k(x:: v s) \\
& \text { in if } p x \\
& \text { then } k^{\prime} \text { nil } \\
& \text { else visit }\left(x s, k^{\prime}\right) \\
& \text { in visit (xs, } \lambda v s . v s) \\
& \text { find_all_prefixes_c } c_{1}(p, x s) \stackrel{\text { def }}{=} \text { letrec visit }(n i l, k) \\
& =\text { nil } \\
& \mid \operatorname{visit}(x:: x s, k) \\
& =\text { let } k^{\prime}=\lambda v s . k(x:: v s) \\
& \text { in if } p x \\
& \text { then }\left(k^{\prime} \text { nil }\right)::\left(\text { visit }\left(x s, k^{\prime}\right)\right) \\
& \text { else visit }\left(x s, k^{\prime}\right) \\
& \text { in visit (xs, גvs.vs) }
\end{aligned}
$$

To find the first prefix, one applies the functional accumulator as soon as a satisfactory list element is found. To list all such prefixes, one continues the traversal, adding the current prefix to the list of the remaining prefixes.

Defunctionalizing these two definitions yields the two definitions of Section 3.1.

The functional accumulator is a delimited continuation:

- In find_first_prefix_c $c_{1}$, visit is written in CPS since all calls are tail calls and all subcomputations are elementary. The continuation is initialized in the initial call to visit, discarded in the base case, extended in the induction case, and used if a satisfactory prefix is found.

- In find_all_prefixes_c $c_{1}$, visit is almost written in CPS except that the continuation is composed if a satisfactory prefix is found: it is used twice - once where it is applied to the empty list to construct a prefix, and once in the visit of the rest of the list to construct a list of prefixes; this prefix is then prepended to the list of prefixes.

These continuation-based programming patterns (initializing a continuation, not using it, or using it more than once as if it were a composable function) have motivated the control operators shift and reset $[27,28]$. Using them, in the next section, we write visit in direct style.

\subsection{Finding prefixes in direct style}

The two following local functions are the direct-style counterpart of the two local functions in Section 3.2: 


$$
\begin{aligned}
& \text { find_first_prefix_c } c_{0}(p, x s) \stackrel{\text { def }}{=} \text { letrec visit nil } \\
& =\mathcal{S k} . \text { nil } \\
& \mid \operatorname{visit}(x:: x s) \\
& =x::(\text { if } p x \text { then nil else visit } x s) \\
& \text { in }\langle\text { visit } x s\rangle \\
& \text { find_all_prefixes_c } c_{0}(p, x s) \stackrel{\text { def }}{=} \text { letrec visit nil } \\
& =\mathcal{S k} \text {.nil } \\
& \text { | visit }(x:: x s) \\
& =x:: \text { if } p x \\
& \text { then } \mathcal{S} k^{\prime} .\left(k^{\prime} \text { nil }\right)::\left\langle k^{\prime}(\text { visit } x s)\right\rangle \\
& \text { in }\langle\text { visit } x s\rangle \\
& \text { else visit } x s
\end{aligned}
$$

In both cases, visit is in direct style, i.e., it is not passed any continuation. The initial calls to visit are enclosed in the control delimiter reset (noted $\langle\cdot\rangle$ for conciseness). In the base cases, the current (delimited) continuation is captured with the control operator shift (noted $\mathcal{S}$ ), which has the effect of emptying the (delimited) context; this captured continuation is bound to an identifier $k$, which is not used; nil is then returned in the emptied context. In the induction case of find_all_prefixes_ $c_{0}$, if the predicate is satisfied, visit captures the current continuation and applies it twice - once to the empty list to construct a prefix, and once to the result of visiting the rest of the list to construct a list of prefixes; this prefix is then prepended to the list of prefixes.

CPS-transforming these two local functions yields the two definitions of Section 3.2 [28].

\subsection{Finding prefixes in continuation-passing style}

The two following local functions are the continuation-passing counterpart of the two local functions in Section 3.2:

$$
\begin{aligned}
& \text { find_first_prefix_c } c_{2}(p, x s) \stackrel{\text { def }}{=} \text { letrec visit }\left(n i l, k_{1}, k_{2}\right) \\
& =k_{2} \text { nil } \\
& \text { | visit }\left(x:: x s, k_{1}, k_{2}\right) \\
& =\text { let } k_{1}^{\prime}=\lambda\left(v s, k_{2}^{\prime}\right) \cdot k_{1}\left(x:: v s, k_{2}^{\prime}\right) \\
& \text { in if } p x \\
& \text { then } k_{1}^{\prime}\left(n i l, k_{2}\right) \\
& \text { else visit }\left(x s, k_{1}^{\prime}, k_{2}\right) \\
& \text { in visit }\left(x s, \lambda\left(v s, k_{2}\right) . k_{2} v s, \lambda v s . v s\right) \\
& \text { find_all_prefixes_c } c_{2}(p, x s) \stackrel{\text { def }}{=} \text { letrec visit }\left(n i l, k_{1}, k_{2}\right) \\
& =k_{2} \text { nil } \\
& \text { | visit ( } \left.x:: x s, k_{1}, k_{2}\right) \\
& =\text { let } k_{1}^{\prime}=\lambda\left(v s, k_{2}^{\prime}\right) \cdot k_{1}\left(x:: v s, k_{2}^{\prime}\right) \\
& \text { in if } p x \\
& \text { then } k_{1}^{\prime}\left(\text { nil, } \lambda v s . v i s i t\left(x s, k_{1}^{\prime}, \lambda v s s . k_{2}(v s:: v s s)\right)\right) \\
& \text { else visit }\left(x s, k_{1}^{\prime}, k_{2}\right) \\
& \text { in visit }\left(x s, \lambda\left(v s, k_{2}\right) . k_{2} \text { vs, } \lambda \text { vss.vss }\right)
\end{aligned}
$$


CPS-transforming the two local functions of Section 3.2 adds another layer of continuations and restores the syntactic characterization of all calls being tail calls and all sub-computations being elementary.

\subsection{The CPS hierarchy}

If $k_{2}$ were used non-tail recursively in a variant of the examples of Section 3.4, we could CPS-transform the definitions one more time, adding one more layer of continuations and restoring the syntactic characterization of all calls being tail calls and all sub-computations being elementary. We could also map this definition back to direct style, eliminating $k_{2}$ but accessing it with shift. If the result were mapped back to direct style one more time, $k_{2}$ would then be accessed with a new control operator, shift 2 , and $k_{1}$ would be accessed with shift (more precisely shift 1 ).

All in all, successive CPS-transformations induce a CPS hierarchy [27,31], and abstracting control up to each successive layer is achieved with successive pairs of control operators shift and reset - reset to initialize the continuation up to a level, and shift to capture a delimited continuation up to this level. Each pair of control operators is indexed by the corresponding level in the hierarchy. Applying a captured continuation packages all the current layers on the next layer and restores the captured layers. When a captured continuation completes, the packaged layers are put back into place and the computation proceeds. (This informal description is made precise in Section 4.)

\subsection{A note about typing}

The type of find_all_prefixes_c $c_{1}$, in Section 3.2, is

$$
(\alpha \rightarrow \text { bool }) \times \alpha \text { list } \rightarrow \alpha \text { list list }
$$

and the type of its local function visit is

$$
\alpha \text { list } \times(\alpha \text { list } \rightarrow \alpha \text { list }) \rightarrow \alpha \text { list list } .
$$

In this example, the co-domain of the continuation is not the same as the co-domain of visit.

Thus find_first_prefix_c $c_{0}$ provides a simple and meaningful example where Filinski's typing of shift [41] does not fit, since it must be used at type

$$
((\beta \rightarrow \text { ans }) \rightarrow \text { ans }) \rightarrow \beta
$$

for a given type ans, i.e., the answer type of the continuation and the type of the computation must be the same. In other words, control effects are not allowed to change the types of the contexts. Due to a similar restriction on the type of shift, the example does not fit either in Murthy's pseudo-classical type system for the CPS hierarchy [62] and in Wadler's most general monadic type system [77, Section 3.4]. It however fits in Danvy and Filinski's original type system [26] which Ariola, Herbelin, and Sabry have recently embedded in classical subtractive $\operatorname{logic}[5]$.

\subsection{Related work}

The example considered in this section builds on the simpler function that unconditionally lists the successive prefixes of a given list. This simpler function is a traditional example of delimited continuations $[20,69]$ : 
- In the Lisp Pointers [20], Danvy presents three versions of this function: a typed continuation-passing version (corresponding to Section 3.2), one with delimited control (corresponding to Section 3.3), and one in assembly language.

- In his PhD thesis [69, Section 6.3], Sitaram presents two versions of this function: one with an accumulator (corresponding to Section 3.1) and one with delimited control (corresponding to Section 3.3).

In Section 3.2, we have shown that the continuation-passing version mediates the version with an accumulator and the version with delimited control since defunctionalizing the continuationpassing version yields one and mapping it back to direct style yields the other.

\subsection{Summary and conclusion}

We have illustrated delimited continuations with the classic example of finding list prefixes, using CPS as a guideline. Direct-style programs using shift and reset can be CPS-transformed into continuation-passing programs where not all calls are tail calls and all sub-computations are elementary. One more CPS transformation establishes this syntactic property with a second layer of continuations. Further CPS transformations provide the extra layers of continuation that are characteristic of the CPS hierarchy.

In the next section, we specify the $\lambda$-calculus extended with shift and reset.

\section{From evaluator to reduction semantics for delimited continuations}

We derive a reduction semantics for the call-by-value $\lambda$-calculus extended with shift and reset, using the method demonstrated in Section 2. First, we transform an evaluator into an environment-based abstract machine. Then we eliminate the environment from this abstract machine, making it substitution-based. Finally, we read all the components of a reduction semantics off the substitution-based abstract machine.

Terms consist of integer literals, variables, $\lambda$-abstractions, function applications, applications of the successor function, reset expressions, and shift expressions:

$$
t::=\ulcorner m\urcorner|x| \lambda x . t\left|t_{0} t_{1}\right| \text { succ } t|\langle t\rangle| \mathcal{S} k . t
$$

Programs are closed terms.

This source language is a subset of the language used in the examples of Section 3. Adding the remaining constructs is a straightforward exercise and does not contribute to our point here.

\subsection{An environment-based evaluator}

Figure 6 displays an evaluator for the language of the first level of the CPS hierarchy. This evaluation function represents the original call-by-value semantics of the $\lambda$-calculus with shift and reset [27], augmented with integer literals and applications of the successor function. It is defined by structural induction over the syntax of terms, and it makes use of an environment $e$, a continuation $k_{1}$, and a meta-continuation $k_{2}$. 
- Terms: term $\ni t::=\ulcorner m\urcorner|x| \lambda x . t\left|t_{0} t_{1}\right|$ succ $t|\langle t\rangle| \mathcal{S} k . t$

- Values: val $\ni v::=m \mid f$

- Answers, meta-continuations, continuations and functions:

$$
\begin{aligned}
\text { ans } & =\text { val } \\
k_{2} \in \text { cont }_{2} & =\text { val } \rightarrow \text { ans } \\
k_{1} \in \text { cont }_{1} & =\text { val } \times \text { cont }_{2} \rightarrow \text { ans } \\
f \in \text { fun } & =\text { val } \times \text { cont }_{1} \times \text { cont }_{2} \rightarrow \text { ans }
\end{aligned}
$$

- Initial continuation and meta-continuation: $\theta_{1}=\lambda\left(v, k_{2}\right) \cdot k_{2} v$

$$
\theta_{2}=\lambda v \cdot v
$$

- Environments: env $\ni e::=e_{\text {empty }} \mid e[x \mapsto v]$

- Evaluation function: eval : term $\times$ env $\times$ cont $_{1} \times$ cont $_{2} \rightarrow$ ans

$$
\begin{aligned}
\text { eval }\left(\ulcorner m\urcorner, e, k_{1}, k_{2}\right)= & k_{1}\left(m, k_{2}\right) \\
\text { eval }\left(x, e, k_{1}, k_{2}\right)= & k_{1}\left(e(x), k_{2}\right) \\
\text { eval }\left(\lambda x . t, e, k_{1}, k_{2}\right)= & k_{1}\left(\lambda\left(v, k_{1}^{\prime}, k_{2}^{\prime}\right) \cdot \text { eval }\left(t, e[x \mapsto v], k_{1}^{\prime}, k_{2}^{\prime}\right), k_{2}\right) \\
\text { eval }\left(t_{0} t_{1}, e, k_{1}, k_{2}\right)= & \text { eval }\left(t_{0}, e, \lambda\left(f, k_{2}^{\prime}\right) . \text { eval }\left(t_{1}, e, \lambda\left(v, k_{2}^{\prime \prime}\right) \cdot f\left(v, k_{1}, k_{2}^{\prime \prime}\right), k_{2}^{\prime}\right), k_{2}\right) \\
\text { eval }\left(\text { succ } t, e, k_{1}, k_{2}\right)= & \text { eval }\left(t, e, \lambda\left(m, k_{2}^{\prime}\right) \cdot k_{1}\left(m+1, k_{2}^{\prime}\right), k_{2}\right) \\
\text { eval }\left(\langle t\rangle, e, k_{1}, k_{2}\right)= & \text { eval }\left(t, e, \theta_{1}, \lambda v \cdot k_{1}\left(v, k_{2}\right)\right) \\
\text { eval }\left(\mathcal{S} k . t, e, k_{1}, k_{2}\right)= & \text { eval }\left(t, e[k \mapsto c], \theta_{1}, k_{2}\right) \\
& \text { where } c=\lambda\left(v, k_{1}^{\prime}, k_{2}^{\prime}\right) \cdot k_{1}\left(v, \lambda v^{\prime} . k_{1}^{\prime}\left(v^{\prime}, k_{2}^{\prime}\right)\right)
\end{aligned}
$$

- Main function: evaluate : term $\rightarrow$ val

$$
\text { evaluate }(t)=\text { eval }\left(t, e_{\text {empty }}, \theta_{1}, \theta_{2}\right)
$$

Figure 6: An environment-based evaluator for the first level of the CPS hierarchy

The evaluation of a terminating program that does not get stuck (i.e., a program where no ill-formed applications occur in the course of evaluation) yields either an integer, a function representing a $\lambda$-abstraction, or a captured continuation. Both evaluate and eval are partial functions to account for non-terminating or stuck programs. The environment stores previously computed values of the free variables of the term under evaluation.

The meta-continuation intervenes to interpret reset expressions and to apply captured continuations. Otherwise, it is passively threaded through the evaluation of literals, variables, $\lambda$-abstractions, function applications, and applications of the successor function. (If it were not for shift and reset, and if eval were curried, $k_{2}$ could be eta-reduced and the evaluator would be in ordinary continuation-passing style.)

The reset control operator is used to delimit control. A reset expression $\langle t\rangle$ is interpreted by evaluating $t$ with the initial continuation and a meta-continuation on which the current continuation has been "pushed." (Indeed, and as will be shown in Section 4.2, defunctionalizing the meta-continuation yields the data type of a stack [29].) 
The shift control operator is used to abstract (delimited) control. A shift expression $\mathcal{S} k . t$ is interpreted by capturing the current continuation, binding it to $k$, and evaluating $t$ in an environment extended with $k$ and with a continuation reset to the initial continuation. Applying a captured continuation is achieved by "pushing" the current continuation on the meta-continuation and applying the captured continuation to the new meta-continuation. Resuming a continuation is achieved by reactivating the "pushed" continuation with the corresponding meta-continuation.

\subsection{An environment-based abstract machine}

The evaluator displayed in Figure 6 is already in continuation-passing style. Therefore, we only need to defunctionalize its expressible values and its continuations to obtain an abstract machine. This abstract machine is displayed in Figure 7 .

The abstract machine consists of three sets of transitions: eval for interpreting terms, cont $_{1}$ for interpreting the defunctionalized continuations (i.e., the evaluation contexts), ${ }^{1}$ and cont $_{2}$ for interpreting the defunctionalized meta-continuations (i.e., the meta-contexts). The set of possible values includes integers, closures and captured contexts. In the original evaluator, the latter two were represented as higher-order functions, but defunctionalizing expressible values of the evaluator has led them to be distinguished.

This eval/apply abstract machine is an extension of the CEK machine [38] with the metacontext $C_{2}$ and its two transitions, and the two transitions for shift and reset. $C_{2}$ intervenes to process reset expressions and to apply captured continuations. Otherwise, it is passively threaded through the processing of literals, variables, $\lambda$-abstractions, function applications, and applications of the successor function. (If it were not for shift and reset, $C_{2}$ and its transitions could be omitted and the abstract machine would reduce to the CEK machine.)

Given an environment $e$, a context $C_{1}$, and a meta-context $C_{2}$, a reset expression $\langle t\rangle$ is processed by evaluating $t$ with the same environment $e$, the empty context $\bullet$, and a metacontext where $C_{1}$ has been pushed on $C_{2}$.

Given an environment $e$, a context $C_{1}$, and a meta-context $C_{2}$, a shift expression $\mathcal{S} k$.t is processed by evaluating $t$ with an extension of $e$ where $k$ denotes $C_{1}$, the empty context [ ], and a meta-context $C_{2}$. Applying a captured context $C_{1}^{\prime}$ is achieved by pushing the current context $C_{1}$ on the current meta-context $C_{2}$ and continuing with $C_{1}^{\prime}$. Resuming a context $C_{1}$ is achieved by popping it off the meta-context $C_{2} \cdot C_{1}$ and continuing with $C_{1}$.

The correctness of the abstract machine with respect to the evaluator is a consequence of the correctness of defunctionalization. In order to express it formally, we define a partial function eval ${ }^{\mathrm{e}}$ mapping a term $t$ to a value $v$ whenever the environment-based machine, started with $t$, stops with $v$. The following theorem states this correctness by relating observable results:

Theorem 1. For any program $t$ and any integer value $m$, evaluate $(t)=m$ if and only if eval $^{\mathrm{e}}(t)=m$.

Proof. The theorem follows directly from the correctness of defunctionalization $[10,63]$.

\footnotetext{
${ }^{1}$ The grammar of evaluation contexts in Figure 7 is isomorphic to the grammar of evaluation contexts in the standard inside-out notation:$$
C_{1}::=[]\left|C_{1}[[](t, e)]\right| C_{1}\left[\text { succ []] } \mid C_{1}[v[]]\right.
$$ 
- Terms: $t::=\ulcorner m\urcorner|x| \lambda x . t\left|t_{0} t_{1}\right|$ succ $t|\langle t\rangle| \mathcal{S} k . t$

- Values (integers, closures, and captured continuations): $v::=m|[x, t, e]| C_{1}$

- Environments: $e::=e_{\text {empty }} \mid e[x \mapsto v]$

- Evaluation contexts: $C_{1}::=[]\left|\operatorname{ARg}\left((t, e), C_{1}\right)\right| \operatorname{sucC}\left(C_{1}\right) \mid \operatorname{Fun}\left(v, C_{1}\right)$

- Meta-contexts: $C_{2}::=\bullet \mid C_{2} \cdot C_{1}$

- Initial transition, transition rules, and final transition:

\begin{aligned}$t & \Rightarrow\left\langle t, e_{\text {empty }},[], \bullet\right\rangle_{\text {eval }} \\$\hline$\left\langle\ulcorner m\urcorner, e, C_{1}, C_{2}\right\rangle_{\text {eval }} & \Rightarrow\left\langle C_{1}, m, C_{2}\right\rangle_{\text {cont }_{1}} \\ \left\langle x, e, C_{1}, C_{2}\right\rangle_{\text {eval }} & \Rightarrow\left\langle C_{1}, e(x), C_{2}\right\rangle_{\text {cont }_{1}} \\ \left\langle\lambda x . t, e, C_{1}, C_{2}\right\rangle_{\text {eval }} & \Rightarrow\left\langle C_{1},[x, t, e], C_{2}\right\rangle_{\text {cont }_{1}} \\ \left\langle t_{0} t_{1}, e, C_{1}, C_{2}\right\rangle_{\text {eval }} & \Rightarrow\left\langle t_{0}, e, \mathrm{ARG}\left(\left(t_{1}, e\right), C_{1}\right), C_{2}\right\rangle_{\text {eval }} \\ \left\langle\text { succ } t, e, C_{1}, C_{2}\right\rangle_{\text {eval }} & \Rightarrow\left\langle t, e, \mathrm{SUCC}\left(C_{1}\right), C_{2}\right\rangle_{\text {eval }} \\ \left\langle\langle t\rangle, e, C_{1}, C_{2}\right\rangle_{\text {eval }} & \Rightarrow\left\langle t, e,[], C_{2} \cdot C_{1}\right\rangle_{\text {eval }} \\ \left\langle\mathcal{S} k . t, e, C_{1}, C_{2}\right\rangle_{\text {eval }} & \Rightarrow\left\langle t, e\left[k \mapsto C_{1}\right],[], C_{2}\right\rangle_{\text {eval }} \\$\hline$\left\langle[], v, C_{2}\right\rangle_{\text {cont }_{1}} & \Rightarrow\left\langle C_{2}, v\right\rangle_{\text {cont }_{2}} \\ \left\langle\mathrm{ARG}\left((t, e), C_{1}\right), v, C_{2}\right\rangle_{\text {cont }_{1}} & \Rightarrow\left\langle t, e, \mathrm{FUN}\left(v, C_{1}\right), C_{2}\right\rangle_{\text {eval }} \\ \left\langle\mathrm{SUCC}\left(C_{1}\right), m, C_{2}\right\rangle_{\text {cont }_{1}} & \Rightarrow\left\langle C_{1}, m+1, C_{2}\right\rangle_{\text {cont }_{1}} \\ \left\langle\mathrm{FUN}\left([x, t, e], C_{1}\right), v, C_{2}\right\rangle_{\text {cont }_{1}} & \Rightarrow\left\langle t, e[x \mapsto v], C_{1}, C_{2}\right\rangle_{\text {eval }} \\ \left\langle\mathrm{FUN}\left(C_{1}^{\prime}, C_{1}\right), v, C_{2}\right\rangle_{\text {cont }_{1}} & \Rightarrow\left\langle C_{1}^{\prime}, v, C_{2} \cdot C_{1}\right\rangle_{\text {cont }_{1}} \\$\hline$\left\langle C_{2} \cdot C_{1}, v\right\rangle_{\text {cont }_{2}} & \Rightarrow\left\langle C_{1}, v, C_{2}\right\rangle_{\text {cont }_{1}} \\$\hline$\langle\bullet, v\rangle_{\text {cont }_{2}} & \Rightarrow v \\$\hline & \end{aligned}

Figure 7: An environment-based abstract machine for the first level of the CPS hierarchy

The environment-based abstract machine can serve both as a foundation for implementing functional languages with control operators for delimited continuations and as a stepping stone in theoretical studies of shift and reset. In the rest of this section, we use it to construct a reduction semantics of shift and reset. 
- Terms and values: $t::=v|x| t_{0} t_{1} \mid$ succ $t|\langle t\rangle| \mathcal{S} k . t$

$v::=\ulcorner m\urcorner|\lambda x . t| C_{1}$

- Evaluation contexts: $C_{1}::=[]\left|\operatorname{ARg}\left(t, C_{1}\right)\right| \operatorname{suCC}\left(C_{1}\right) \mid \operatorname{FUN}\left(v, C_{1}\right)$

- Meta-contexts: $C_{2}::=\bullet \mid C_{2} \cdot C_{1}$

- Initial transition, transition rules, and final transition:

\begin{tabular}{|c|c|c|}
\hline$t$ & $\Rightarrow$ & $\langle t,[], \bullet\rangle_{\text {eval }}$ \\
\hline$\left\langle\ulcorner m\urcorner, C_{1}, C_{2}\right\rangle_{\text {eval }}$ & $\Rightarrow$ & $\left\langle C_{1},\ulcorner m\urcorner, C_{2}\right\rangle_{\text {cont }_{1}}$ \\
\hline$\left\langle\lambda x . t, C_{1}, C_{2}\right\rangle_{e v a l}$ & $\Rightarrow$ & $\left\langle C_{1}, \lambda x . t, C_{2}\right\rangle_{\text {cont }_{1}}$ \\
\hline$\left\langle C_{1}^{\prime}, C_{1}, C_{2}\right\rangle_{\text {eval }}$ & $\Rightarrow$ & $\left\langle C_{1}, C_{1}^{\prime}, C_{2}\right\rangle_{\text {cont }_{1}}$ \\
\hline$\left\langle t_{0} t_{1}, C_{1}, C_{2}\right\rangle_{e v a l}$ & $\Rightarrow$ & $\left\langle t_{0}, \operatorname{ARG}\left(t_{1}, C_{1}\right), C_{2}\right\rangle_{\text {eval }}$ \\
\hline$\left\langle\text { succ } t, C_{1}, C_{2}\right\rangle_{\text {eval }}$ & $\Rightarrow$ & $\left\langle t, \operatorname{SuCC}\left(C_{1}\right), C_{2}\right\rangle_{\text {eval }}$ \\
\hline$\left\langle\langle t\rangle, C_{1}, C_{2}\right\rangle_{\text {eval }}$ & $\Rightarrow$ & $\left\langle t,[], C_{2} \cdot C_{1}\right\rangle_{\text {eval }}$ \\
\hline$\left\langle\mathcal{S} k . t, C_{1}, C_{2}\right\rangle_{\text {eval }}$ & $\Rightarrow$ & $\left\langle t\left\{C_{1} / k\right\},[], C_{2}\right\rangle_{\text {eval }}$ \\
\hline$\left\langle[], v, C_{2}\right\rangle_{\text {cont }_{1}}$ & $\Rightarrow$ & $\left\langle C_{2}, v\right\rangle_{\text {cont }_{2}}$ \\
\hline$\left\langle\operatorname{ARG}\left(t, C_{1}\right), v, C_{2}\right\rangle_{\text {cont }_{1}}$ & $\Rightarrow$ & $\left\langle t, \operatorname{FUN}\left(v, C_{1}\right), C_{2}\right\rangle_{\text {eval }}$ \\
\hline$\left\langle\operatorname{SUCC}\left(C_{1}\right),\ulcorner m\urcorner, C_{2}\right\rangle_{\text {cont }_{1}}$ & $\Rightarrow$ & $\left\langle C_{1},\ulcorner m+1\urcorner, C_{2}\right\rangle_{\text {cont }_{1}}$ \\
\hline$\left\langle\operatorname{FUN}\left(\lambda x . t, C_{1}\right), v, C_{2}\right\rangle_{\text {cont }_{1}}$ & $\Rightarrow$ & $\left\langle t\{v / x\}, C_{1}, C_{2}\right\rangle_{\text {eval }}$ \\
\hline$\left\langle\operatorname{FUN}\left(C_{1}^{\prime}, C_{1}\right), v, C_{2}\right\rangle_{\text {cont }_{1}}$ & $\Rightarrow$ & $\left\langle C_{1}^{\prime}, v, C_{2} \cdot C_{1}\right\rangle_{\text {cont }_{1}}$ \\
\hline$\left\langle C_{2} \cdot C_{1}, v\right\rangle_{\text {cont }_{2}}$ & $\Rightarrow$ & $\left\langle C_{1}, v, C_{2}\right\rangle_{\text {cont }_{1}}$ \\
\hline$\langle\bullet, v\rangle_{\text {cont }_{2}}$ & $\Rightarrow$ & $v$ \\
\hline
\end{tabular}

Figure 8: A substitution-based abstract machine for the first level of the CPS hierarchy

\subsection{A substitution-based abstract machine}

The environment-based abstract machine of Figure 7, on which we want to base our development, makes a distinction between terms and values. Since a reduction semantics is specified by purely syntactic operations (it gives meaning to terms by specifying their rewriting strategy and an appropriate notion of reduction, and is indeed also referred to as 'syntactic theory'), we need to embed the domain of values back into the syntax. To this end we transform the environment-based abstract machine into the substitution-based abstract machine displayed in Figure 8. The transformation is standard, except that we also need to embed evaluation contexts in the syntax; hence the substitution-based machine operates on terms where "quoted" (in the sense of Lisp) contexts can occur. (If it were not for shift and reset, 
$C_{2}$ and its transitions could be omitted and the abstract machine would reduce to the $\mathrm{CK}$ machine [38].)

We write $t\{v / x\}$ to denote the result of the usual capture-avoiding substitution of the value $v$ for $x$ in $t$.

Formally, the relationship between the two machines is expressed with the following simulation theorem, where evaluation with the substitution-based abstract machine is captured by the partial function eval ${ }^{\mathrm{s}}$, defined analogously to eval ${ }^{\mathrm{e}}$.

Theorem 2. For any program $t$ and any values $v, v^{\prime}$, eval $\left.\right|^{\mathbf{s}}(t)=v$ if and only if eval ${ }^{\mathbf{e}}(t)=v^{\prime}$ and $\mathcal{T}\left(v^{\prime}\right)=v$. The function $\mathcal{T}$ relates a semantic value with its syntactic representation and is defined as follows: ${ }^{2}$

$$
\begin{aligned}
\mathcal{T}(m) & =\ulcorner m\urcorner \\
\mathcal{T}([x, t, e]) & =\lambda x . t\left\{\mathcal{T}\left(e\left(x_{1}\right)\right) / x_{1}\right\} \ldots\left\{\mathcal{T}\left(e\left(x_{n}\right)\right) / x_{n}\right\},\left\{x_{1}, \ldots, x_{n}\right\}=F V(\lambda x . t) \\
\mathcal{T}([]) & =[] \\
\mathcal{T}\left(\operatorname{ARG}\left((t, e), C_{1}\right)\right) & =\operatorname{ARG}\left(t\left\{\mathcal{T}\left(e\left(x_{1}\right)\right) / x_{1}\right\} \ldots\left\{\mathcal{T}\left(e\left(x_{n}\right)\right) / x_{n}\right\}, \mathcal{T}\left(C_{1}\right)\right),\left\{x_{1}, \ldots, x_{n}\right\}=F V(t) \\
\mathcal{T}\left(\operatorname{FUN}\left(v, C_{1}\right)\right) & =\operatorname{FUn}\left(\mathcal{T}(v), \mathcal{T}\left(C_{1}\right)\right) \\
\mathcal{T}\left(\operatorname{SUCC}\left(C_{1}\right)\right) & =\operatorname{SUCC}\left(\mathcal{T}\left(C_{1}\right)\right)
\end{aligned}
$$

Proof. We extend the translation function $\mathcal{T}$ to meta-contexts and configurations, in the expected way, e.g.,

$$
\begin{aligned}
& \mathcal{T}\left(\left\langle t, e, C_{1}, C_{2}\right\rangle_{\text {eval }}\right)=\left\langle t\left\{\mathcal{T}\left(e\left(x_{1}\right)\right) / x_{1}\right\} \ldots\left\{\mathcal{T}\left(e\left(x_{n}\right)\right) / x_{n}\right\}, \mathcal{T}\left(C_{1}\right), \mathcal{T}\left(C_{2}\right)\right\rangle_{\text {eval }} \\
& \text { where }\left\{x_{1}, \ldots, x_{n}\right\}=F V(t)
\end{aligned}
$$

Then it is straightforward to show that the two abstract machines operate in lock step with respect to the translation. Hence, for any program $t$, both machines diverge or they both stop (after the same number of transitions) with the values $v$ and $\mathcal{T}(v)$, respectively.

We now proceed to analyze the transitions of the machine displayed in Figure 8. We can think of a configuration $\left\langle t, C_{1}, C_{2}\right\rangle_{\text {eval }}$ as the following decomposition of the initial term into a meta-context $C_{2}$, a context $C_{1}$, and an intermediate term $t$ :

$$
C_{2} \# C_{1}[t]
$$

where \# separates the context and the meta-context. Each transition performs either a reduction, or a decomposition in search of the next redex. Let us recall that a decomposition is performed when both sides of a transition are partitions of the same term; in that case, depending on the structure of the decomposition $C_{2} \# C_{1}[t]$, a subpart of the term is chosen to be evaluated next, and the contexts are updated accordingly. We also observe that evaltransitions follow the structure of $t$, cont $t_{1}$-transitions follow the structure of $C_{1}$ when the term has been reduced to a value, and cont $t_{2}$-transitions follow the structure of $C_{2}$ when a value in the empty context has been reached.

Next we specify all the components of the reduction semantics based on the analysis of the abstract machine.

\footnotetext{
${ }^{2} \mathcal{T}$ is a generalization of Plotkin's function Real [64].
} 


\subsection{A reduction semantics}

A reduction semantics provides a reduction relation on expressions by defining values, evaluation contexts, and redexes $[35,37,38,79]$. In the present case,

- the values are already specified in the (substitution-based) abstract machine:

$$
v::=\ulcorner m\urcorner|\lambda x . t| C_{1}
$$

- the evaluation contexts and meta-contexts are already specified in the abstract machine, as the data-type part of defunctionalized continuations;

$$
\begin{aligned}
& C_{1}::=[]\left|\operatorname{ARG}\left(t, C_{1}\right)\right| \operatorname{FUn}\left(v, C_{1}\right) \mid \operatorname{sucC}\left(C_{1}\right) \\
& C_{2}::=\bullet \mid C_{2} \cdot C_{1}
\end{aligned}
$$

- we can read the redexes off the transitions of the abstract machine:

$$
r::=\operatorname{succ}\ulcorner m\urcorner|(\lambda x . t) v| \mathcal{S} k . t\left|C_{1}^{\prime} v\right|\langle v\rangle
$$

Based on the distinction between decomposition and reduction, we single out the following reduction rules from the transitions of the machine:

$$
\begin{array}{lrl}
(\delta) & C_{2} \# C_{1}[\text { succ }\ulcorner m\urcorner] & \rightarrow C_{2} \# C_{1}[\ulcorner m+1\urcorner] \\
\left(\beta_{\lambda}\right) & C_{2} \# C_{1}[(\lambda x . t) v] & \rightarrow C_{2} \# C_{1}[t\{v / x\}] \\
\left(\mathcal{S}_{\lambda}\right) & C_{2} \# C_{1}[\mathcal{S} k . t] & \rightarrow C_{2} \#\left[t\left\{C_{1} / k\right\}\right] \\
\left(\beta_{c t x}\right) & C_{2} \# C_{1}\left[C_{1}^{\prime} v\right] & \rightarrow C_{2} \cdot C_{1} \# C_{1}^{\prime}[v] \\
\text { (val) } & C_{2} \# C_{1}[\langle v\rangle] & \rightarrow C_{2} \# C_{1}[v]
\end{array}
$$

$\left(\beta_{\lambda}\right)$ is the usual call-by-value $\beta$-reduction; we have renamed it to indicate that the applied term is a $\lambda$-abstraction, since we can also apply a captured context, as in $\left(\beta_{c t x}\right) .\left(\mathcal{S}_{\lambda}\right)$ is plausibly symmetric to $\left(\beta_{\lambda}\right)$ - it can be seen as an application of the abstraction $\lambda k$.t to the current context. Moreover, $\left(\beta_{c t x}\right)$ can be seen as performing both a reduction and a decomposition: it is a reduction because an application of a context with a hole to a value is reduced to the value plugged into the hole; and it is a decomposition because it changes the meta-context, as if the application were enclosed in a reset. Finally, (val) makes it possible to pass the boundary of a context when the term inside this context has been reduced to a value.

The $\beta_{c t x}$-rule and the $\mathcal{S}_{\lambda}$-rule give a justification for representing a captured context $C_{1}$ as a term $\lambda x \cdot\left\langle C_{1}[x]\right\rangle$, as found in other studies of shift and reset $[53,54,62]$. In particular, the need for delimiting the captured context is a consequence of the $\beta_{c t x}$-rule.

Finally, we can read the decomposition function off the transitions of the abstract machine:

$$
\begin{aligned}
& \text { decompose }(t)=\text { decompose }^{\prime}(t,[], \bullet) \\
& \text { decompose' }\left(t_{0} t_{1}, C_{1}, C_{2}\right)=\text { decompose }^{\prime}\left(t_{0}, \operatorname{ARG}\left(t_{1}, C_{1}\right), C_{2}\right) \\
& \text { decompose }\left(\text { succ } t, C_{1}, C_{2}\right)=\text { decompose }^{\prime}\left(t, \operatorname{SUCC}\left(C_{1}\right), C_{2}\right) \\
& \text { decompose }^{\prime}\left(\langle t\rangle, C_{1}, C_{2}\right)=\text { decompose }^{\prime}\left(t,[], C_{2} \cdot C_{1}\right) \\
& \text { decompose }^{\prime}\left(v, \operatorname{ARG}\left(t, C_{1}\right), C_{2}\right)=\text { decompose }^{\prime}\left(t, \operatorname{FUN}\left(v, C_{1}\right), C_{2}\right)
\end{aligned}
$$


In the remaining cases either a value or a redex has been found:

$$
\begin{aligned}
\text { decompose }^{\prime}(v,[], \bullet) & =\bullet \#[v] \\
\text { decompose }^{\prime}\left(v,[], C_{2} \cdot C_{1}\right) & =C_{2} \# C_{1}[\langle v\rangle] \\
\text { decompose }^{\prime}\left(\mathcal{S} k . t, C_{1}, C_{2}\right) & =C_{2} \# C_{1}[\mathcal{S} k . t] \\
\text { decompose }^{\prime}\left(v, \operatorname{FUN}\left((\lambda x . t), C_{1}\right), C_{2}\right) & =C_{2} \# C_{1}[(\lambda x . t) v] \\
\text { decompose }^{\prime}\left(v, \operatorname{FUN}\left(C_{1}^{\prime}, C_{1}\right), C_{2}\right) & =C_{2} \# C_{1}\left[C_{1}^{\prime} v\right] \\
\text { decompose }^{\prime}\left(\ulcorner m\urcorner, \operatorname{SUCC}\left(C_{1}\right), C_{2}\right) & =C_{2} \# C_{1}[\operatorname{succ}\ulcorner m\urcorner]
\end{aligned}
$$

An inverse of the decompose function, traditionally called plug, reconstructs a term from its decomposition:

$$
\begin{aligned}
\operatorname{plug}(\bullet \#[t]) & =t \\
\operatorname{plug}\left(C_{2} \cdot C_{1} \#[t]\right) & =\operatorname{plug}\left(C_{2} \# C_{1}[\langle t\rangle]\right) \\
\operatorname{plug}\left(C_{2} \#\left(\operatorname{ARG}\left(t^{\prime}, C_{1}\right)\right)[t]\right) & =\operatorname{plug}\left(C_{2} \# C_{1}\left[t t^{\prime}\right]\right) \\
\operatorname{plug}\left(C_{2} \#\left(\operatorname{FUN}\left(v, C_{1}\right)\right)[t]\right) & =p \operatorname{lug}\left(C_{2} \# C_{1}[v t]\right) \\
\operatorname{plug}\left(C_{2} \#\left(\operatorname{SUCC}\left(C_{1}\right)\right)[t]\right) & =\operatorname{plug}\left(C_{2} \# C_{1}[\operatorname{succ} t]\right)
\end{aligned}
$$

In order to talk about unique decomposition, we need to define the set of potential redexes (i.e., the disjoint union of actual redexes and stuck redexes). The grammar of potential redexes reads as follows:

$$
p::=\operatorname{succ} v\left|v_{0} v_{1}\right| \mathcal{S} k . t \mid\langle v\rangle
$$

Lemma 1 (Unique decomposition). A program $t$ is either a value $v$ or there exist a unique context $C_{1}$, a unique meta-context $C_{2}$ and a potential redex $p$ such that $t=p l u g\left(C_{2} \# C_{1}[p]\right)$. In the former case decompose $(t)=\bullet \#[v]$ and in the latter case either decompose $(t)=$ $C_{2} \# C_{1}[p]$ if $p$ is an actual redex, or decompose $(t)$ is undefined.

Proof. The first part follows by induction on the structure of $t$. The second part follows from the equation decompose $\left(p \operatorname{lug}\left(C_{2} \# C_{1}[r]\right)\right)=C_{2} \# C_{1}[r]$ which holds for all $C_{2}, C_{1}$ and $r$.

It is evident that evaluating a program either using the derived reduction rules or using the substitution-based abstract machine yields the same result.

Theorem 3. For any program $t$ and any value $v$, eval ${ }^{\mathbf{s}}(t)=v$ if and only if $t \rightarrow^{*} v$, where $\rightarrow^{*}$ is the reflexive, transitive closure of the one-step reduction defined by the relation $\rightarrow$.

Proof. When evaluating with the abstract machine, each contraction is followed by decomposing the contractum in the current context and meta-context. When evaluating with the reduction rules, however, each contraction is followed by plugging the contractum and decomposing the resulting term. Therefore, the theorem follows from the equation

$$
\text { decompose }^{\prime}\left(t, C_{1}, C_{2}\right)=\operatorname{decompose}\left(\operatorname{plug}\left(C_{2} \# C_{1}[t]\right)\right)
$$

which holds for any $C_{2}, C_{1}$ and $t$.

We have verified that using refocusing [30], one can go from this reduction semantics to the abstract machine of Figure 8. 


\subsection{Beyond CPS}

Alternatively to using the meta-context to compose delimited continuations, as in Figure 7, we could compose them by concatenating their representation. Such a concatenation function is defined as follows:

$$
\begin{aligned}
{[] \star C_{1}^{\prime} } & =C_{1}^{\prime} \\
\left(\operatorname{ARG}\left((t, e), C_{1}\right)\right) \star C_{1}^{\prime} & =\operatorname{ARG}\left((t, e), C_{1} \star C_{1}^{\prime}\right) \\
\left(\operatorname{SUCC}\left(C_{1}\right)\right) \star C_{1}^{\prime} & =\operatorname{sUCC}\left(C_{1} \star C_{1}^{\prime}\right) \\
\left(\operatorname{FUN}\left(v, C_{1}\right)\right) \star C_{1}^{\prime} & =\operatorname{FUn}\left(v, C_{1} \star C_{1}^{\prime}\right)
\end{aligned}
$$

(The second clause would read $\left(\operatorname{ARG}\left(t, C_{1}\right)\right) \star C_{1}^{\prime}=\operatorname{ARG}\left(t, C_{1} \star C_{1}^{\prime}\right)$ for the contexts of Figure 8.)

Then, in Figures 7 and 8, we could replace the transition

$$
\left\langle\mathrm{FUN}\left(C_{1}^{\prime}, C_{1}\right), v, C_{2}\right\rangle_{\text {cont }_{1}} \Rightarrow\left\langle C_{1}^{\prime}, v, C_{2} \cdot C_{1}\right\rangle_{\text {cont }_{1}}
$$

by the following one:

$$
\left\langle\operatorname{FUN}\left(C_{1}^{\prime}, C_{1}\right), v, C_{2}\right\rangle_{\text {cont }_{1}} \Rightarrow\left\langle C_{1}^{\prime} \star C_{1}, v, C_{2}\right\rangle_{\text {cont }_{1}}
$$

This replacement changes the control effect of shift to that of Felleisen et al.'s $\mathcal{F}$ operator [36]. Furthermore, the modified abstract machine is in structural correspondence with Felleisen et al.'s abstract machine for $\mathcal{F}$ and \# $[36,40]$.

This representation of control (as a list of 'stack frames') and this implementation of composing delimited continuations (by concatenating these lists) are at the heart of virtually all non-CPS-based accounts of delimited control. However, the modified environment-based abstract machine does not correspond to a defunctionalized continuation-passing evaluator because it is not in the range of defunctionalization [29] since the first-order representation of functions should have a single point of consumption. Here, the constructors of contexts are not solely consumed by the cont $_{1}$ transitions of the abstract machine as in Figures 7 and 8 , but also by $\star$. Therefore, the abstract machine that uses $\star$ is not in the range of Reynolds's defunctionalization and it thus does not immediately correspond to a higher-order, continuation-passing evaluator. In that sense, control operators using $\star$ go beyond CPS.

Elsewhere [18], we have rephrased the modified abstract machine to put it in defunctionalized form, and we have exhibited the corresponding higher-order evaluator and the corresponding continuation-passing style. This dynamic CPS is not just plain CPS but is a form of continuation+state-passing style where the threaded state is a list of intermediate delimited continuations. Unexpectedly, it is also in structural correspondence with the architecture for delimited control recently proposed by Dybvig, Peyton Jones, and Sabry on other operational grounds [34].

In any case, and irrespectively of any new dynamic CPS and any new architecture for delimited control, there seems to be remarkably few examples that actually illustrate the expressive power of dynamic delimited continuations. We have recently presented one, breadth-first traversal [17], and we present another one below. 
The two following functions traverse a given list and return another list. The recursive call to visit is abstracted into a delimited continuation, which is applied to the tail of the list:

$$
\begin{aligned}
& \text { foo } x s \stackrel{\text { def }}{=} \text { letrec visit nil bar xs } \stackrel{\text { def }}{=} \text { letrec visit nil } \\
& =\text { nil } \\
& \text { | visit ( } x:: x s) \\
& =\operatorname{visit}(\mathcal{S} k . x::(k x s)) \\
& \text { in 〈visit xs }\rangle \\
& \begin{array}{c}
\text { bar } x s \stackrel{\text { def }}{=} \text { letrec visit nil } \\
=\text { nil } \\
\mid \text { visit }(x:: x s) \\
=\text { visit }(\mathcal{F} k . x::(k x s)) \\
\text { in }\langle\text { visit } x s\rangle
\end{array}
\end{aligned}
$$

On the left, foo uses $\mathcal{S}$ and on the right, bar uses $\mathcal{F}$; for the rest, the two definitions are identical. Given an input list, foo copies it and bar reverses it.

To explain this difference and to account for the extended source language, we need to expand the grammar of evaluation contexts, e.g., with a production to account for calls to the list constructor:

$$
C_{1}::=[]\left|\operatorname{ARg}\left(t, C_{1}\right)\right| \operatorname{succ}\left(C_{1}\right)\left|\operatorname{Fun}\left(v, C_{1}\right)\right| \operatorname{CONS}\left(v, C_{1}\right) \mid \ldots
$$

Similarly, we need to expand the definition of concatenation as follows:

$$
\left(\operatorname{Cons}\left(v, C_{1}\right)\right) \star C_{1}^{\prime}=\operatorname{Cons}\left(v, C_{1} \star C_{1}^{\prime}\right)
$$

Here is a trace of the two computations in the form of the calls to and returns from visit for the input list $1:: 2::$ nil:

foo: Every time the captured continuation is resumed, its representation is kept separate from the current context. The meta-context therefore grows whereas the captured context solely consists of FUN(visit, []) throughout (writing visit in the context for simplicity):

$$
\begin{aligned}
C_{2} & \# C_{1}[\langle\text { visit }(1:: 2:: \text { nil })\rangle] \\
C_{2} \cdot C_{1} & \#[\text { visit }(1:: 2:: \text { nil })] \\
C_{2} \cdot C_{1} \cdot(\operatorname{CoNS}(1,[])) & \#[\text { visit }(2:: \text { nil })] \\
C_{2} \cdot C_{1} \cdot(\operatorname{CoNS}(1,[])) \cdot(\operatorname{CoNS}(2,[])) & \#[\text { visit nil }] \\
C_{2} \cdot C_{1} \cdot(\operatorname{CoNS}(1,[])) \cdot(\operatorname{CoNS}(2,[])) & \#[\text { nil }] \\
C_{2} \cdot C_{1} \cdot(\operatorname{CoNS}(1,[])) & \#[2:: \text { nil }] \\
C_{2} \cdot C_{1} & \#[1:: 2:: \text { nil }] \\
C_{2} & \# C_{1}[1:: 2:: \text { nil }]
\end{aligned}
$$

bar: Every time the captured continuation is resumed, its representation is concatenated to the current context. The meta-context therefore remains the same whereas the context changes dynamically. The first captured context is FUN(visit, []); concatenating it to $\operatorname{CONS}(1,[])$ yields CONS $(1, \mathrm{FUN}($ visit, []$))$, which is the second captured context:

$$
\begin{aligned}
C_{2} & \# C_{1}[\langle\text { visit }(1:: 2:: \text { nil })\rangle] \\
C_{2} \cdot C_{1} & \#[\text { visit }(1:: 2:: \text { nil })] \\
C_{2} \cdot C_{1} & \#(\operatorname{CONS}(1,[]))[\text { visit }(2:: \text { nil })] \\
C_{2} \cdot C_{1} & \#(\operatorname{CONS}(1, \operatorname{CONS}(2,[])))[\text { visit nil }] \\
C_{2} \cdot C_{1} & \#(\operatorname{CONS}(1, \operatorname{CONS}(2,[])))[\text { nil }] \\
C_{2} \cdot C_{1} & \#(\operatorname{CONS}(2,[]))[1:: \text { nil }] \\
C_{2} \cdot C_{1} & \#[2:: 1:: \text { nil] }] \\
C_{2} & \# C_{1}[2:: 1:: \text { nil }]
\end{aligned}
$$




\subsection{Summary and conclusion}

We have presented the original evaluator for the $\lambda$-calculus with shift and reset; this evaluator uses two layers of continuations. From this call-by-value evaluator we have derived two abstract machines, an environment-based one and a substitution-based one; each of these machines uses two layers of evaluation contexts. Based on the substitution-based machine we have constructed a reduction semantics for the $\lambda$-calculus with shift and reset; this reduction semantics, by construction, is sound with respect to CPS.

\section{$5 \quad$ From evaluator to reduction semantics for the CPS hierarchy}

We construct a reduction semantics for the call-by-value $\lambda$-calculus extended with shift $_{n}$ and $\operatorname{reset}_{n}$. As in Section 4, we go from an evaluator to an environment-based abstract machine, and from a substitution-based abstract machine to a reduction semantics. Because of the regularity of CPS, the results can be generalized from level 1 to higher levels without repeating the actual construction, based only on the original specification of the hierarchy [27]. In particular, the proofs of the theorems generalize straightforwardly from level 1.

\subsection{An environment-based evaluator}

At the $n$th level of the hierarchy, the language is extended with operators shift $_{i}$ and reset $_{i}$ for all $i$ such that $1 \leq i \leq n$. The evaluator for this language is shown in Figures 9 and 10. If $n=1$, it coincides with the evaluator displayed in Figure 6 .

- Terms $(1 \leq i \leq n)$ : term $\ni t::=\ulcorner m\urcorner|x| \lambda x . t\left|t_{0} t_{1}\right|$ succ $t\left|\langle t\rangle_{i}\right| \mathcal{S}_{i} k . t$

- Values: val $\ni v::=m \mid f$

- Answers, continuations and functions $(1 \leq i \leq n)$ :

$$
\begin{aligned}
\text { ans } & =\text { val } \\
k_{n+1} \in \operatorname{cont}_{n+1} & =\text { val } \rightarrow \text { ans } \\
k_{i} \in \text { cont }_{i} & =\text { val } \times \operatorname{cont}_{i+1} \times \ldots \times \text { cont }_{n+1} \rightarrow \text { ans } \\
f \in \text { fun } & =\text { val } \times \operatorname{cont}_{1} \times \ldots \times \text { cont }_{n+1} \rightarrow \text { ans }
\end{aligned}
$$

- Initial continuations $(1 \leq i \leq n)$ :

$$
\begin{aligned}
\theta_{i} & =\lambda\left(v, k_{i+1}, k_{i+2}, \ldots, k_{n+1}\right) \cdot k_{i+1}\left(v, k_{i+2}, \ldots, k_{n+1}\right) \\
\theta_{n+1} & =\lambda v \cdot v
\end{aligned}
$$

- Environments: env $\ni e::=e_{\text {empty }} \mid e[x \mapsto v]$

- Evaluation function: see Figure 10

Figure 9: An environment-based evaluator for the CPS hierarchy at level $n$ 
- Evaluation function $(1 \leq i \leq n)$ : eval $_{n}:$ term $\times$ env $\times$ cont $_{1} \times \ldots \times$ cont $_{n+1} \rightarrow$ ans

$\operatorname{eval}_{n}\left(\ulcorner m\urcorner, e, k_{1}, k_{2}, \ldots, k_{n+1}\right)=k_{1}\left(m, k_{2}, \ldots, k_{n+1}\right)$

$\operatorname{eval}_{n}\left(x, e, k_{1}, k_{2}, \ldots, k_{n+1}\right)=k_{1}\left(e(x), k_{2}, \ldots, k_{n+1}\right)$

$\operatorname{eval}_{n}\left(\lambda x . t, e, k_{1}, k_{2}, \ldots, k_{n+1}\right)=k_{1}\left(\lambda\left(v, k_{1}^{\prime}, k_{2}^{\prime}, \ldots, k_{n+1}^{\prime}\right)\right.$. eval $\left._{n}\left(t, e[x \mapsto v], k_{1}^{\prime}, k_{2}^{\prime}, \ldots, k_{n+1}^{\prime}\right), k_{2}, \ldots, k_{n+1}\right)$

$\mathrm{eval}_{n}\left(t_{0} t_{1}, e, k_{1}, k_{2}, \ldots, k_{n+1}\right)=\operatorname{eval}_{n}\left(t_{0}, e\right.$,

$$
\begin{aligned}
\lambda\left(f, k_{2}^{\prime}, \ldots, k_{n+1}^{\prime}\right) \cdot \operatorname{eval}_{n} & \left(t_{1}, e,\right. \\
& \lambda\left(v, k_{2}^{\prime \prime}, \ldots, k_{n+1}^{\prime \prime}\right) . f\left(v, k_{1}, k_{2}^{\prime \prime}, \ldots, k_{n+1}^{\prime \prime}\right), \\
& \left.k_{2}^{\prime}, \ldots, k_{n+1}^{\prime}\right),
\end{aligned}
$$

$$
\left.k_{2}, \ldots, k_{n+1}\right)
$$

$\operatorname{eval}_{n}\left(\operatorname{succ} t, e, k_{1}, k_{2}, \ldots, k_{n+1}\right)=\operatorname{eval}_{n}\left(t, e, \lambda\left(m, k_{2}^{\prime}, \ldots, k_{n+1}^{\prime}\right) . k_{1}\left(m+1, k_{2}^{\prime}, \ldots, k_{n+1}^{\prime}\right), k_{2}, \ldots, k_{n+1}\right)$

$\operatorname{eval}_{n}\left(\langle t\rangle_{i}, e, k_{1}, k_{2}, \ldots, k_{n+1}\right)=\operatorname{eval}_{n}\left(t, e, \theta_{1}, \ldots, \theta_{i}, \lambda\left(v, k_{i+2}^{\prime}, \ldots, k_{n+1}^{\prime}\right) . k_{1}\left(v, k_{2}, \ldots, k_{i}, k_{i+1}, k_{i+2}^{\prime}, \ldots, k_{n+1}^{\prime}\right), k_{i+2}, \ldots, k_{n+1}\right)$ $\operatorname{eval}_{n}\left(\mathcal{S}_{i} k . t, e, k_{1}, k_{2}, \ldots, k_{n+1}\right)=\operatorname{eval}_{n}\left(t, e\left[k \mapsto c_{i}\right], \theta_{1}, \ldots, \theta_{i}, k_{i+1}, \ldots, k_{n+1}\right)$

where $c_{i}=\lambda\left(v, k_{1}^{\prime}, \ldots, k_{n+1}^{\prime}\right) . k_{1}\left(v, k_{2}, \ldots, k_{i}, \lambda\left(v^{\prime}, k_{i+2}^{\prime \prime}, \ldots, k_{n+1}^{\prime \prime}\right) . k_{1}^{\prime}\left(v^{\prime}, k_{2}^{\prime}, \ldots, k_{i+1}^{\prime}, k_{i+2}^{\prime \prime}, \ldots, k_{n+1}^{\prime \prime}\right), k_{i+2}^{\prime}, \ldots, k_{n+1}^{\prime}\right)$

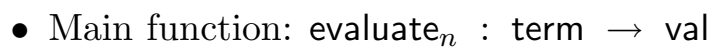

evaluate $_{n}(t)=\operatorname{eval}_{n}\left(t, e_{\text {empty }}, \theta_{1}, \ldots, \theta_{n}, \theta_{n+1}\right)$

Figure 10: An environment-based evaluator for the CPS hierarchy at level $n$, ctd. 
The evaluator uses $n+1$ layers of continuations. In the five first clauses (literal, variable, $\lambda$ abstraction, function application, and application of the successor function), the continuations $k_{2}, \ldots, k_{n+1}$ are passive: if the evaluator were curried, they could be eta-reduced. In the clauses defining $\operatorname{shift}_{i}$ and $\operatorname{reset}_{i}$, the continuations $k_{i+2}, \ldots, k_{n+1}$ are also passive. Each pair of control operators is indexed by the corresponding level in the hierarchy: reset $_{i}$ is used to "push" each successive continuation up to level $i$ onto level $i+1$ and to reinitialize them with $\theta_{1}, \ldots, \theta_{i}$, which are the successive CPS counterparts of the identity function; shift $_{i}$ is used to abstract control up to level $i$ into a delimited continuation and to reinitialize the successive continuations up to level $i$ with $\theta_{1}, \ldots, \theta_{i}$.

Applying a delimited continuation that was abstracted up to level $i$ "pushes" each successive continuation up to level $i$ onto level $i+1$ and restores the successive continuations that were captured in a delimited continuation. When such a delimited continuation completes, and when an expression delimited by reset $_{i}$ completes, the successive continuations that were pushed onto level $i+1$ are "popped" back into place and the computation proceeds.

\subsection{An environment-based abstract machine}

Defunctionalizing the evaluator of Figures 9 and 10 yields the environment-based abstract machine displayed in Figures 11 and 12. If $n=1$, it coincides with the abstract machine displayed in Figure 7.

The abstract machine consists of $n+2$ sets of transitions: eval for interpreting terms and cont $_{1}, \ldots$, cont $_{n+1}$ for interpreting the successive defunctionalized continuations. The set of possible values includes integers, closures and captured contexts.

This abstract machine is an extension of the abstract machine displayed in Figure 7 with $n+1$ contexts instead of 2 and the corresponding transitions for $\operatorname{shift}_{i}$ and reset $_{i}$. Each meta $_{i+1}$-context intervenes to process reset ${ }_{i}$ expressions and to apply captured continuations. Otherwise, the successive contexts are passively threaded to process literals, variables, $\lambda$ abstractions, function applications, and applications of the successor function.

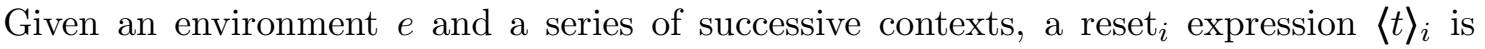
processed by evaluating $t$ with the same environment $e, i$ empty contexts, and a meta ${ }_{i+1^{-}}$ context over which all the intermediate contexts have been pushed on.

- Terms $(1 \leq i \leq n): \quad t::=\ulcorner m\urcorner|x| \lambda x . t\left|t_{0} t_{1}\right|$ succ $t\left|\langle t\rangle_{i}\right| \mathcal{S}_{i} k . t$

- Values $(1 \leq i \leq n): \quad v::=m|[x, t, e]| C_{i}$

- Evaluation contexts $(2 \leq i \leq n+1)$ :

$$
\begin{aligned}
& C_{1}::=[]\left|\operatorname{ARG}\left((t, e), C_{1}\right)\right| \operatorname{succ}\left(C_{1}\right) \mid \operatorname{FUN}\left(v, C_{1}\right) \\
& C_{i}::=[] \mid C_{i} \cdot C_{i-1}
\end{aligned}
$$

- Environments: $e::=e_{\text {empty }} \mid e[x \mapsto v]$

- Initial transition, transition rules, and final transition: see Figure 12

Figure 11: An environment-based abstract machine for the CPS hierarchy at level $n$ 
- Initial transition, transition rules, and final transition $(1 \leq i \leq n, 2 \leq j \leq n)$ :

\begin{tabular}{|c|c|c|}
\hline$t$ & $\Rightarrow$ & $\left\langle t, e_{\text {empty }},[],[], \ldots,[]\right\rangle_{\text {eval }}$ \\
\hline $\begin{array}{r}\left\langle\ulcorner m\urcorner, e, C_{1}, C_{2}, \ldots, C_{n+1}\right\rangle_{\text {eval }} \\
\left\langle x, e, C_{1}, C_{2}, \ldots, C_{n+1}\right\rangle_{\text {eval }} \\
\left\langle\lambda x . t, e, C_{1}, C_{2}, \ldots, C_{n+1}\right\rangle_{\text {eval }} \\
\left\langle t_{0} t_{1}, e, C_{1}, C_{2}, \ldots, C_{n+1}\right\rangle_{\text {eval }} \\
\left\langle\text { succ } t, e, C_{1}, C_{2}, \ldots, C_{n+1}\right\rangle_{\text {eval }} \\
\left\langle\langle t\rangle_{i}, e, C_{1}, C_{2}, \ldots, C_{n+1}\right\rangle_{\text {eval }} \\
\left\langle\mathcal{S}_{i} k . t, e, C_{1}, C_{2}, \ldots, C_{n+1}\right\rangle_{\text {eval }}\end{array}$ & $\begin{array}{l}\Rightarrow \\
\Rightarrow \\
\Rightarrow \\
\Rightarrow \\
\Rightarrow \\
\Rightarrow\end{array}$ & $\begin{array}{l}\left\langle C_{1}, m, C_{2}, \ldots, C_{n+1}\right\rangle_{\text {cont }_{1}} \\
\left\langle C_{1}, e(x), C_{2}, \ldots, C_{n+1}\right\rangle_{\text {cont }_{1}} \\
\left\langle C_{1},[x, t, e], C_{2}, \ldots, C_{n+1}\right\rangle_{\text {cont }_{1}} \\
\left\langle t_{0}, e, \operatorname{ARG}\left(\left(t_{1}, e\right), C_{1}\right), C_{2}, \ldots, C_{n+1}\right\rangle_{\text {eval }} \\
\left\langle t, e, \operatorname{SUCC}\left(C_{1}\right), C_{2}, \ldots, C_{n+1}\right\rangle_{\text {eval }} \\
\left\langle t, e,[], \ldots,[], C_{i+1} \cdot\left(C_{i} \cdot \ldots\left(C_{2} \cdot C_{1}\right) \ldots\right), C_{i+2}, \ldots, C_{n+1}\right\rangle_{\text {eval }} \\
\left\langle t, e\left[k \mapsto C_{i} \cdot\left(C_{i-1} \cdot \ldots\left(C_{2} \cdot C_{1}\right) \ldots\right)\right],[], \ldots,[], C_{i+1}, \ldots, C_{n+1}\right\rangle_{\text {eval }}\end{array}$ \\
\hline $\begin{array}{r}\left\langle[], v, C_{2}, \ldots, C_{n+1}\right\rangle_{\text {cont }_{1}} \\
\left\langle\operatorname{ARG}\left((t, e), C_{1}\right), v, C_{2}, \ldots, C_{n+1}\right\rangle_{\text {cont }_{1}} \\
\left\langle\operatorname{SUCC}\left(C_{1}\right), m, C_{2}, \ldots, C_{n+1}\right\rangle_{\text {cont }_{1}} \\
\left\langle\operatorname{FUN}\left([x, t, e], C_{1}\right), v, C_{2}, \ldots, C_{n+1}\right\rangle_{\text {cont }_{1}} \\
\left\langle\operatorname{FUN}\left(\left(C_{i}^{\prime} \cdot\left(C_{i-1}^{\prime} \cdot \ldots\left(C_{2}^{\prime} \cdot C_{1}^{\prime}\right) \ldots\right)\right), C_{1}\right), v, C_{2}, \ldots, C_{n+1}\right\rangle_{\text {cont }_{1}}\end{array}$ & $\begin{array}{l}\Rightarrow \\
\Rightarrow \\
\Rightarrow \\
\Rightarrow\end{array}$ & $\begin{array}{l}\left\langle C_{2}, v, C_{3}, \ldots, C_{n+1}\right\rangle_{\text {cont }_{2}} \\
\left\langle t, e, \operatorname{FUN}\left(v, C_{1}\right), C_{2}, \ldots, C_{n+1}\right\rangle_{\text {eval }} \\
\left\langle C_{1}, m+1, C_{2}, \ldots, C_{n+1}\right\rangle_{\text {cont }_{1}} \\
\left\langle t, e[x \mapsto v], C_{1}, C_{2}, \ldots, C_{n+1}\right\rangle_{\text {eval }} \\
\left\langle C_{1}^{\prime}, v, C_{2}^{\prime}, \ldots, C_{i}^{\prime}, C_{i+1} \cdot\left(C_{i} \cdot \ldots\left(C_{2} \cdot C_{1}\right) \ldots\right), C_{i+2}, \ldots, C_{n+1}\right\rangle_{\text {cont }_{1}}\end{array}$ \\
\hline $\begin{array}{r}\left\langle[], v, C_{j+1}, \ldots, C_{n+1}\right\rangle_{\text {cont }_{j}} \\
\left\langle C_{j} \cdot\left(C_{j-1} \cdot \ldots\left(C_{2} \cdot C_{1}\right) \ldots\right), v, C_{j+1}, \ldots, C_{n+1}\right\rangle_{\text {cont }_{j}}\end{array}$ & $\begin{array}{l}\Rightarrow \\
\Rightarrow\end{array}$ & $\begin{array}{l}\left\langle C_{j+1}, v, C_{j+2}, \ldots, C_{n+1}\right\rangle_{\text {cont }_{j+1}} \\
\left\langle C_{1}, v, C_{2}, \ldots, C_{n+1}\right\rangle_{\text {cont }_{1}}\end{array}$ \\
\hline$\left\langle C_{n+1} \cdot\left(C_{n} \cdot \ldots\left(C_{2} \cdot C_{1}\right) \ldots\right), v\right\rangle_{\text {cont }_{n+1}}$ & $\Rightarrow$ & $\left\langle C_{1}, v, C_{2}, \ldots, C_{n+1}\right\rangle_{\text {cont }_{1}}$ \\
\hline$\langle[], v\rangle_{\text {cont }_{n+1}}$ & $\rightarrow$ & $v$ \\
\hline
\end{tabular}

Figure 12: An environment-based abstract machine for the CPS hierarchy at level $n$, ctd. 
Given an environment $e$ and a series of successive contexts, a shift expression $\mathcal{S}_{i} k . t$ is processed by evaluating $t$ with an extension of $e$ where $k$ denotes a composition of the $i$ surrounding contexts, $i$ empty contexts, and the remaining outer contexts. Applying a captured context is achieved by pushing all the current contexts on the next outer context, restoring the composition of the captured contexts, and continuing with them. Resuming a composition of captured contexts is achieved by popping them off the next outer context and continuing with them.

In order to relate the resulting abstract machine to the evaluator, we define a partial function evale mapping a term $t$ to a value $v$ whenever the machine for level $n$, started with $t$, stops with $v$. The correctness of the machine with respect to the evaluator is ensured by the following theorem:

Theorem 4. For any program $t$ and any integer value $m$, evaluate ${ }_{n}(t)=m$ if and only if $\mathrm{eval}_{n}^{\mathrm{e}}(t)=m$.

\subsection{A substitution-based abstract machine}

In the same fashion as in Section 4.3, we construct the substitution-based abstract machine corresponding to the environment-based abstract machine of Section 5.2. The result is displayed in Figures 13 and 14. If $n=1$, it coincides with the abstract machine displayed in Figure 8.

The $n$th level contains $n+1$ evaluation contexts and each context $C_{i}$ can be viewed as a stack of non-empty contexts $C_{i-1}$. Terms are decomposed as

$$
C_{n+1} \#_{n} C_{n} \#_{n-1} C_{n-1} \#_{n-2} \cdots \#_{2} C_{2} \#_{1} C_{1}[t],
$$

where each $\#_{i}$ represents a context delimiter of level $i$. All the control operators that occur at the $j$ th level (with $j<n$ ) of the hierarchy do not use the contexts $j+2, \ldots, n+1$. The functions decompose and its inverse plug can be read off the machine, as for level 1.

The transitions of the machine for level $j$ are "embedded" in the machine for level $j+1$; the extra components are threaded but not used.

We define a partial function eval ${ }_{n}^{\mathrm{s}}$ capturing the evaluation by the substitution-based abstract machine for an arbitrary level $n$, analogously to the definition of evale ${ }_{n}^{\mathrm{e}}$. Now we can

- Terms and values $(1 \leq i \leq n): \quad t::=v|x| t_{0} t_{1} \mid$ succ $t\left|\langle t\rangle_{i}\right| \mathcal{S}_{i} k . t$

$$
v::=\ulcorner m\urcorner|\lambda x . t| C_{i}
$$

- Evaluation contexts $(2 \leq i \leq n+1)$ :

$$
\begin{aligned}
& C_{1}::=[]\left|\operatorname{ARG}\left(t, C_{1}\right)\right| \operatorname{sucC}\left(C_{1}\right) \mid \operatorname{FUN}\left(v, C_{1}\right) \\
& C_{i}::=[] \mid C_{i} \cdot C_{i-1}
\end{aligned}
$$

- Initial transition, transition rules, and final transition: see Figure 14

Figure 13: A substitution-based abstract machine for the CPS hierarchy at level $n$ 
- Initial transition, transition rules, and final transition $(1 \leq i \leq n, 2 \leq j \leq n)$ :

\begin{tabular}{|c|c|c|}
\hline$t$ & $\Rightarrow$ & $\langle t,[],[], \ldots,[]\rangle_{\text {eval }}$ \\
\hline $\begin{array}{r}\left\langle\ulcorner m\urcorner, C_{1}, C_{2}, \ldots, C_{n+1}\right\rangle_{\text {eval }} \\
\left\langle\lambda x . t, C_{1}, C_{2}, \ldots, C_{n+1}\right\rangle_{\text {eval }} \\
\left\langle C_{i}^{\prime}, C_{1}, C_{2}, \ldots, C_{n+1}\right\rangle_{\text {eval }} \\
\left\langle t_{0} t_{1}, C_{1}, C_{2}, \ldots, C_{n+1}\right\rangle_{\text {eval }} \\
\left\langle\text { succ } t, C_{1}, C_{2}, \ldots, C_{n+1}\right\rangle_{\text {eval }} \\
\left\langle\mathcal{S}_{i} k . t, C_{1}, C_{2}, \ldots, C_{n+1}\right\rangle_{\text {eval }} \\
\left\langle\langle t\rangle_{i}, C_{1}, C_{2}, \ldots, C_{n+1}\right\rangle_{\text {eval }}\end{array}$ & $\begin{array}{l}\Rightarrow \\
\Rightarrow \\
\Rightarrow \\
\Rightarrow \\
\Rightarrow \\
\Rightarrow\end{array}$ & $\begin{array}{l}\left\langle C_{1},\ulcorner m\urcorner, C_{2}, \ldots, C_{n+1}\right\rangle_{\text {cont }_{1}} \\
\left\langle C_{1}, \lambda x . t, C_{2}, \ldots, C_{n+1}\right\rangle_{\text {cont }_{1}} \\
\left\langle C_{1}, C_{i}^{\prime}, C_{2}, \ldots, C_{n+1}\right\rangle_{\text {cont }_{1}} \\
\left\langle t_{0}, \operatorname{ARG}\left(\left(t_{1}, e\right), C_{1}\right), C_{2}, \ldots, C_{n+1}\right\rangle_{\text {eval }} \\
\left\langle t, \operatorname{suCC}\left(C_{1}\right), C_{2}, \ldots, C_{n+1}\right\rangle_{\text {eval }} \\
\left\langle t\left\{C_{i} \cdot\left(C_{i-1} \cdot \ldots\left(C_{2} \cdot C_{1}\right) \ldots\right) / k\right\},[], \ldots,[], C_{i+1}, \ldots, C_{n+1}\right\rangle_{\text {eval }} \\
\left\langle t,[], \ldots,[], C_{i+1} \cdot\left(C_{i} \cdot \ldots\left(C_{2} \cdot C_{1}\right) \ldots\right), C_{i+2}, \ldots, C_{n+1}\right\rangle_{\text {eval }}\end{array}$ \\
\hline $\begin{array}{r}\left\langle[], v, C_{2}, \ldots, C_{n+1}\right\rangle_{\text {cont }_{1}} \\
\left\langle\operatorname{ARG}\left(t, C_{1}\right), v, C_{2}, \ldots, C_{n+1}\right\rangle_{\text {cont }_{1}} \\
\left\langle\operatorname{SUCC}\left(C_{1}\right),\ulcorner m\urcorner, C_{2}, \ldots, C_{n+1}\right\rangle_{\text {cont }_{1}} \\
\left\langle\operatorname{FUN}\left((\lambda x . t), C_{1}\right), v, C_{2}, \ldots, C_{n+1}\right\rangle_{\text {cont }_{1}} \\
\left\langle\operatorname{FUN}\left(\left(C_{i}^{\prime} \cdot\left(C_{i-1}^{\prime} \cdot \ldots\left(C_{2}^{\prime} \cdot C_{1}^{\prime}\right) \ldots\right)\right), C_{1}\right), v, C_{2}, \ldots, C_{n+1}\right\rangle_{\text {cont }_{1}}\end{array}$ & $\begin{array}{l}\Rightarrow \\
\Rightarrow \\
\Rightarrow \\
\Rightarrow\end{array}$ & $\begin{array}{l}\left\langle C_{2}, v, C_{3}, \ldots, C_{n+1}\right\rangle_{\text {cont }_{2}} \\
\left\langle t, \operatorname{FUN}\left(v, C_{1}\right), C_{2}, \ldots, C_{n+1}\right\rangle_{\text {eval }} \\
\left\langle C_{1},\ulcorner m+1\urcorner, C_{2}, \ldots, C_{n+1}\right\rangle_{\text {cont }_{1}} \\
\left\langle t\{v / x\}, C_{1}, C_{2}, \ldots, C_{n+1}\right\rangle_{\text {eval }} \\
\left\langle C_{1}^{\prime}, v, C_{2}^{\prime}, \ldots, C_{i}^{\prime}, C_{i+1} \cdot\left(C_{i} \cdot \ldots\left(C_{2} \cdot C_{1}\right) \ldots\right), C_{i+2}, \ldots, C_{n+1}\right\rangle_{\text {cont }_{1}}\end{array}$ \\
\hline $\begin{array}{r}\left\langle[], v, C_{j+1}, \ldots, C_{n+1}\right\rangle_{\text {cont }_{j}} \\
\left\langle C_{j} \cdot\left(C_{j-1} \cdot \ldots\left(C_{2} \cdot C_{1}\right) \ldots\right), v, C_{j+1}, \ldots, C_{n+1}\right\rangle_{\text {cont }_{j}}\end{array}$ & $\begin{array}{l}\Rightarrow \\
\Rightarrow\end{array}$ & $\begin{array}{l}\left\langle C_{j+1}, v, C_{j+2}, \ldots, C_{n+1}\right\rangle_{\text {cont }_{j+1}} \\
\left\langle C_{1}, v, C_{2}, \ldots, C_{n+1}\right\rangle_{\text {cont }_{1}}\end{array}$ \\
\hline$\left\langle C_{n+1} \cdot\left(C_{n} \cdot \ldots\left(C_{2} \cdot C_{1}\right) \ldots\right), v\right\rangle_{\text {cont }_{n+1}}$ & $\Rightarrow$ & $\left\langle C_{1}, v, C_{2}, \ldots, C_{n+1}\right\rangle_{\text {cont }_{1}}$ \\
\hline$\langle[], v\rangle_{\text {cont }_{n+1}}$ & $\Rightarrow$ & $v$ \\
\hline
\end{tabular}

Figure 14: A substitution-based abstract machine for the the CPS hierarchy at level $n$, ctd. 
relate evaluation with the environment-based and the substitution-based abstract machines for level $n$.

Theorem 5. For any program $t$ and any values $v, v^{\prime}$ in the language of level $n$, eval $\left.\right|_{n} ^{\mathbf{s}}(t)=v$ if and only if eval ${ }_{n}^{\mathrm{e}}(t)=v^{\prime}$ and $\mathcal{T}_{n}\left(v^{\prime}\right)=v$.

The definition of $\mathcal{T}_{n}$ extends that of $\mathcal{T}$ from Theorem 2 in such a way that it is homomorphic for all the contexts $C_{i}$, with $2 \leq i \leq n$.

\subsection{A reduction semantics}

Along the same lines as in Section 4.4, we construct the reduction semantics for the CPS hierarchy based on the abstract machine of Figures 13 and 14. For an arbitrary level $n$ we obtain the following set of reduction rules, for all $1 \leq i \leq n$; they define the actual redexes:

$$
C_{n+1} \#_{n} \cdots \#_{1} C_{1}[\operatorname{succ}\ulcorner m\urcorner] \rightarrow_{n} C_{n+1} \#_{n} \cdots \#_{1} C_{1}[\ulcorner m+1\urcorner]
$$

$$
C_{n+1} \#_{n} \cdots \#_{1} C_{1}[(\lambda x . t) v] \rightarrow_{n} C_{n+1} \#_{n} \cdots \#_{1} C_{1}[t\{v / x\}]
$$

$$
C_{n+1} \#_{n} \cdots \#_{1} C_{1}\left[\mathcal{S}_{i} k . t\right] \rightarrow_{n}
$$

$$
C_{n+1} \#_{n} \ldots \#_{i+1} C_{i+1} \#_{i}[] \ldots \#_{1}\left[t\left\{C_{i} \cdot\left(\ldots\left(C_{2} \cdot C_{1}\right) \ldots\right) / k\right\}\right]
$$

$\left(\beta_{c t x}^{i}\right) \quad C_{n+1} \#_{n} \cdots \#_{1} C_{1}\left[C_{i}^{\prime} \cdot\left(\ldots\left(C_{2}^{\prime} \cdot C_{1}^{\prime}\right) \ldots\right) v\right] \rightarrow_{n}$

$$
C_{n+1} \#_{n} \cdots \#_{i+1} C_{i+1} \cdot\left(C_{i} \cdot\left(\ldots\left(C_{2} \cdot C_{1}\right) \ldots\right)\right) \#_{i} C_{i}^{\prime} \#_{i-1} \cdots \#_{1} C_{1}^{\prime}[v]
$$

$$
C_{n+1} \#_{n} \cdots \#_{1} C_{1}\left[\langle v\rangle_{i}\right] \rightarrow_{n} C_{n+1} \#_{n} \cdots \#_{1} C_{1}[v]
$$

Each level contains all the reductions from lower levels, and these reductions are compatible with additional layers of evaluation contexts. In particular, at level 0 there are only $\delta$ and $\beta_{\lambda}$-reductions.

The values and evaluation contexts are already specified in the abstract machine. Moreover, the potential redexes are defined according to the following grammar:

$$
p_{n}::=\operatorname{succ} v\left|v_{0} v_{1}\right| \mathcal{S}_{i} k . t \mid\langle v\rangle_{i} \quad(1 \leq i \leq n)
$$

Lemma 2 (Unique decomposition for level $n$ ). A program $t$ is either a value or there exists a unique sequence of contexts $C_{1}, \ldots, C_{n+1}$ and a potential redex $p_{n}$ such that $t=\operatorname{plug}\left(C_{n+1} \#_{n} \cdots \#_{1} C_{1}\left[p_{n}\right]\right)$.

Evaluating a term using either the derived reduction rules or the substitution-based abstract machine from Section 5.3 yields the same result:

Theorem 6. For any program $t$ and any value $v$, eval $\left.\right|_{n} ^{\mathrm{s}}(t)=v$ if and only if $t \rightarrow_{n}^{*} v$, where $\rightarrow_{n}^{*}$ is the reflexive, transitive closure of $\rightarrow_{n}$.

As in Section 4.4, using refocusing, one can go from a given reduction semantics of Section 5.4 into a pre-abstract machine and the corresponding eval/apply abstract machine of Figures 13 and 14.

\subsection{Beyond CPS}

As in Section 4.5, one can define a concatenation function over contexts and use it to implement composable continuations in the CPS hierarchy. Again the modified environment-based abstract machine does not immediately correspond to a defunctionalized continuation-passing evaluator. Such control operators go beyond traditional CPS. 


\subsection{Summary and conclusion}

We have generalized the results presented in Section 4 from level 1 to the whole CPS hierarchy of control operators shift ${ }_{n}$ and reset $_{n}$. Starting from the original evaluator for the $\lambda$-calculus with $\operatorname{shift}_{n}$ and reset $_{n}$ that uses $n+1$ layers of continuations, we have derived two abstract machines, an environment-based one and a substitution-based one; each of these machines use $n+1$ layers of evaluation contexts. Based on the substitution-based machine we have obtained a reduction semantics for the $\lambda$-calculus extended with $\operatorname{shift}_{n}$ and reset $_{n}$ which, by construction, is sound with respect to CPS.

\section{Programming in the CPS hierarchy}

To finish, we present new examples of programming in the CPS hierarchy. The examples are normalization functions. In Sections 6.1 and 6.2, we first describe normalization by evaluation and we present the simple example of the free monoid. In Section 6.3, we present a function mapping a proposition into its disjunctive normal form; this normalization function uses delimited continuations. In Section 6.4, we generalize the normalization functions of Sections 6.2 and 6.3 to a hierarchical language of units and products, and we express the corresponding normalization function in the CPS hierarchy.

\subsection{Normalization by evaluation}

Normalization by evaluation is a 'reduction-free' approach to normalizing terms. Instead of reducing a term to its normal form, one evaluates this term into a non-standard model and reifies its denotation into its normal form [33]:

$$
\begin{aligned}
\text { eval } & : \text { term } \rightarrow \text { value }^{\mathrm{nf}} \\
\text { reify } & : \text { value } \rightarrow \text { term }^{\mathrm{nf}} \\
\text { normalize } & : \text { term } \rightarrow \text { term }^{\mathrm{nf}} \\
\text { normalize } & =\text { reify } \circ \text { eval }
\end{aligned}
$$

Normalization by evaluation has been developed in intuitionistic type theory [19,59], proof theory $[12,13]$, category theory [4], and partial evaluation [21,22], where it has emerged as a new field of application for delimited continuations [9, 22, 33, 43, 46, 49, 74].

\subsection{The free monoid}

A source term in the free monoid is either a variable, the unit element, or the product of two terms:

$$
\text { term } \ni t::=x|\varepsilon| t \star t^{\prime}
$$

The product is associative and the unit element is neutral. These properties justify the following conversion rules:

$$
\begin{aligned}
t \star\left(t^{\prime} \star t^{\prime \prime}\right) & \leftrightarrow\left(t \star t^{\prime}\right) \star t^{\prime \prime} \\
t \star \varepsilon & \leftrightarrow t \\
\varepsilon \star t & \leftrightarrow t
\end{aligned}
$$


We aim (for example) for list-like flat normal forms:

$$
\text { term }^{\mathrm{nf}} \ni \widehat{t}::=\varepsilon^{\mathrm{nf}} \mid x \star^{\mathrm{nf}} \widehat{t}
$$

In a reduction-based approach to normalization, one would orient the conversion rules into reduction rules and one would apply these reduction rules until a normal form is obtained:

$$
\begin{aligned}
t \star\left(t^{\prime} \star t^{\prime \prime}\right) & \leftarrow\left(t \star t^{\prime}\right) \star t^{\prime \prime} \\
\varepsilon \star t & \rightarrow t
\end{aligned}
$$

In a reduction-free approach to normalization, one defines a normalization function as the composition of a non-standard evaluation function and a reification function. Let us state such a normalization function.

The non-standard domain of values is the transformer

$$
\text { value }=\text { term }^{\mathrm{nf}} \rightarrow \text { term }^{\mathrm{nf}} .
$$

The evaluation function is defined by induction over the syntax of source terms, and the reification function inverts it:

$$
\begin{aligned}
\text { eval } x & =\lambda t . x \star^{\mathrm{nf}} t \\
\text { eval } \varepsilon & =\lambda t . t \\
\text { eval }\left(t \star t^{\prime}\right) & =(\text { eval } t) \circ\left(\text { eval } t^{\prime}\right) \\
\text { reify } v & =v \varepsilon^{\mathrm{nf}} \\
\text { normalize } t & =\text { reify }(\text { eval } t)
\end{aligned}
$$

In effect, eval is a mapping from the source monoid to the monoid of transformers (unit is mapped to unit and products are mapped to products) and the normalization function hinges on the built-in associativity of function composition. Dybjer et al. have studied its theoretical content $[14,19,56]$. From a (functional) programming standpoint, the reductionbased approach amounts to flattening a tree iteratively by reordering it, and the reduction-free approach amounts to flattening a tree with an accumulator.

\subsection{A language of propositions}

A source term, i.e., a proposition, is either a variable, a literal (true or false), a conjunction, or a disjunction:

$$
\text { term } \ni t::=x \mid \text { true }\left|t \wedge t^{\prime}\right| \text { false } \mid t \vee t^{\prime}
$$

Conjunction and disjunction are associative and distribute over each other; true is neutral for conjunction and absorbant for disjunction; and false is neutral for disjunction and absorbant for conjunction.

We aim (for example) for list-like disjunctive normal forms:

$$
\begin{aligned}
& \text { term }^{\mathrm{nf}} \ni \widehat{t}::=d \\
& \text { term }_{\mathrm{d}}^{\mathrm{nf}} \ni d::=\text { false }^{\mathrm{nf}} \mid c \vee^{\mathrm{nf}} d \\
& \text { term }_{\mathrm{c}}^{\mathrm{nf}} \ni c::=\text { true }^{\mathrm{nf}} \mid x \wedge{ }^{\mathrm{nf}} c
\end{aligned}
$$

Our normalization function is the result of composing a non-standard evaluation function and a reification function. We state them below without proof. 
Given the domains of transformers

$$
\begin{aligned}
& F_{1}=t e r m_{\mathrm{c}}^{\mathrm{nf}} \rightarrow t_{\text {erm }}^{\mathrm{nf}} \\
& F_{2}=\text { term }_{\mathrm{d}}^{\mathrm{nf}} \rightarrow \text { term }_{\mathrm{d}}^{\mathrm{nf}}
\end{aligned}
$$

the non-standard domain of values is ans $_{1}$, where

$$
\begin{aligned}
\text { ans }_{2} & =F_{2} \\
\text { ans }_{1} & =\left(F_{1} \rightarrow a n s_{2}\right) \rightarrow a n s_{2} .
\end{aligned}
$$

The evaluation function is defined by induction over the syntax of source terms, and the reification function inverts it:

$$
\begin{aligned}
& \text { eval }_{0} x k d=k\left(\lambda c . x \wedge^{\mathrm{nf}} c\right) d \\
& \text { eval }_{0} \text { true } k d=k(\lambda c . c) d \\
& \operatorname{eval}_{0}\left(t \wedge t^{\prime}\right) k d=\text { eval }_{0} t\left(\lambda f_{1} \cdot \text { eval }_{0} t^{\prime}\left(\lambda f_{1}^{\prime} \cdot k\left(f_{1} \circ f_{1}^{\prime}\right)\right)\right) d \\
& \text { eval } \text { false } \mathrm{d}=d \\
& \operatorname{eval}_{0}\left(t \vee t^{\prime}\right) k d=\operatorname{eval}_{0} t k\left(\operatorname{eval}_{0} t^{\prime} k d\right) \\
& \text { reify } y_{0} v=v\left(\lambda f_{1} \cdot \lambda d \cdot\left(f_{1} \text { true }^{\mathrm{nf}}\right) \vee^{\mathrm{nf}} d\right) \text { false }^{\mathrm{nf}} \\
& \text { normalize } t=\text { reify }_{0}\left(\text { eval }_{0} t\right)
\end{aligned}
$$

This normalization function uses a continuation $k$, an accumulator $d$ to flatten disjunctions, and another one $c$ to flatten conjunctions. The continuation is delimited: the three first clauses of $e^{2} \mathrm{l}_{0}$ are in CPS; in the fourth, $k$ is discarded (accounting for the fact that false is absorbant for conjunction); and in the last, $k$ is duplicated and used in non-tail position (achieving the distribution of conjunctions over disjunctions). The continuation and the accumulators are initialized in the definition of reif $y_{0}$.

Uncurrying the continuation and mapping eval $l_{0}$ and reify $y_{0}$ back to direct style yield the following definition, which lives at level 1 of the CPS hierarchy:

$$
\begin{aligned}
\text { eval }_{1} x d= & \left(\lambda c . x \wedge^{\mathrm{nf}} c, d\right) \\
\text { eval }_{1} \text { true } d= & (\lambda c . c, d) \\
\text { eval }_{1}\left(t \wedge t^{\prime}\right) d= & \text { let }\left(f_{1}, d\right)=\text { eval }_{1} t d \\
& \text { in let }\left(f_{1}^{\prime}, d\right)=\text { eval }_{1} t^{\prime} d \\
& \quad \text { in }\left(f_{1} \circ f_{1}^{\prime}, d\right) \\
\text { eval }_{1} \text { false } d== & \text { Sk.d } \\
\text { eval }_{1}\left(t \vee t^{\prime}\right) d= & \mathcal{S} k . k\left(\text { eval }_{1} t\left\langle k\left(\text { eval }_{1} t^{\prime} d\right)\right\rangle\right) \\
\text { reify }_{1} v= & \left\langle\text { let }\left(f_{1}, d\right)=v \text { false }^{\mathrm{nf}}\right. \\
& \text { in } \left.\left(f_{1} \text { true }^{\mathrm{nf}}\right) \vee^{\mathrm{nf}} d\right\rangle \\
\text { normalize } t= & \text { reify }_{1}\left(\text { eval }_{1} t\right)
\end{aligned}
$$

The three first clauses of $e^{2} l_{1}$ are in direct style; the two others abstract control with shift. In the fourth clause, the context is discarded; and in the last clause, the context is duplicated and composed. The context and the accumulators are initialized in the definition of reify ${ }_{1}$.

This direct-style version makes it even more clear than the CPS version that the accumulator for the disjunctions in normal form is a threaded state. A continuation-based, state-based version (or better, a monad-based one) can therefore be written-but it is out of scope here. 


\subsection{A hierarchical language of units and products}

We consider a generalization of propositional logic where a source term is either a variable, a unit in a hierarchy of units, or a product in a hierarchy of products:

$$
\begin{aligned}
\text { term } \ni t::= & x\left|\varepsilon_{i}\right| t \star_{i} t^{\prime} \\
& \text { where } 1 \leq i \leq n .
\end{aligned}
$$

All the products are associative. All units are neutral for products with the same index.

The free monoid: The language corresponds to that of the free monoid if $n=1$, as in Section 6.2.

Boolean logic: The language corresponds to that of propositions if $n=2$, as in Section 6.3: $\varepsilon_{1}$ is true, $\star_{1}$ is $\wedge, \varepsilon_{2}$ is false, and $\star_{2}$ is $\vee$.

Multi-valued logic: In general, for each $n>2$ we can consider a suitable $n$-valued logic [45]; for example, in case $n=4$, the language corresponds to that of Belnap's bilattice $\mathcal{F O U R}$ [11]. It is also possible to modify the normalization function to work for less regular logical structures (e.g., other bilattices).

Monads: In general, the language corresponds to that of layered monads [60]: each unit element is the unit of the corresponding monad, and each product is the 'bind' of the corresponding monad. In practice, layered monads are collapsed into one for programming consumption [42], but prior to this collapse, all the individual monad operations coexist in the computational soup.

In the remainder of this section, we assume that all the products, besides being associative, distribute over each other, and that all units, besides being neutral for products with the same index, are absorbant for products with other indices. We aim (for example) for a generalization of disjunctive normal forms:

$$
\begin{aligned}
& \text { term }^{\mathrm{nf}} \ni \widehat{t}::=t_{n} \\
& \operatorname{term}_{n}^{\mathrm{nf}} \ni t_{n}::=\varepsilon_{n}^{\mathrm{nf}} \mid t_{n-1} \star_{n}^{\mathrm{nf}} t_{n} \\
& \vdots \\
& \text { term }_{1}^{\mathrm{nf}} \ni t_{1}::=\varepsilon_{1}^{\mathrm{nf}} \mid t_{0} \star_{1}^{\mathrm{nf}} t_{1} \\
& \text { term }_{0}^{\mathrm{nf}} \ni t_{0}::=x
\end{aligned}
$$

For presentational reasons, in the remainder of this section we arbitrarily fix $n$ to be 5 .

Our normalization function is the result of composing a non-standard evaluation function and a reification function. We state them below without proof. Given the domains of transformers

$$
\begin{aligned}
& F_{1}=\text { term }_{1}^{\text {nf }} \rightarrow \text { term }_{1}^{\text {nf }} \\
& F_{2}=\text { term }_{2}^{\text {nf }} \rightarrow \text { term }_{2}^{\text {nf }} \\
& F_{3}=\text { term }_{3}^{\text {nf }} \rightarrow \text { term }_{3}^{\text {nf }} \\
& F_{4}=\text { term }_{4}^{\text {nf }} \rightarrow \text { term }_{4}^{\text {nf }} \\
& F_{5}=\text { term }_{5}^{\text {nf }} \rightarrow \text { term }_{5}^{\text {nf }}
\end{aligned}
$$

the non-standard domain of values is ans 1 , where 


$$
\begin{aligned}
\text { ans }_{5} & =F_{5} \\
\text { ans }_{4} & =\left(F_{4} \rightarrow \text { ans }_{5}\right) \rightarrow \text { ans }_{5} \\
\text { ans }_{3} & =\left(F_{3} \rightarrow \text { ans }_{4}\right) \rightarrow \text { ans }_{4} \\
\text { ans }_{2} & =\left(F_{2} \rightarrow \text { ans }_{3}\right) \rightarrow \text { ans }_{3} \\
\text { ans }_{1} & =\left(F_{1} \rightarrow \text { ans }_{2}\right) \rightarrow \text { ans }_{2} .
\end{aligned}
$$

The evaluation function is defined by induction over the syntax of source terms, and the reification function inverts it:

$$
\begin{aligned}
& \operatorname{eval}_{0} x k_{1} k_{2} k_{3} k_{4} t_{5}=k_{1}\left(\lambda t_{1} \cdot x \star_{1}^{\mathrm{nf}} t_{1}\right) k_{2} k_{3} k_{4} t_{5} \\
& \mathrm{eval}_{0} \varepsilon_{1} k_{1} k_{2} k_{3} k_{4} t_{5}=k_{1}\left(\lambda t_{1} \cdot t_{1}\right) k_{2} k_{3} k_{4} t_{5} \\
& \operatorname{eval}_{0}\left(t \star_{1} t^{\prime}\right) k_{1} k_{2} k_{3} k_{4} t_{5}=\operatorname{eval}_{0} t\left(\lambda f_{1} . \operatorname{eval}_{0} t^{\prime}\left(\lambda f_{1}^{\prime} \cdot k_{1}\left(f_{1} \circ f_{1}^{\prime}\right)\right)\right) k_{2} k_{3} k_{4} t_{5} \\
& \mathrm{eval}_{0} \varepsilon_{2} k_{1} k_{2} k_{3} k_{4} t_{5}=k_{2}\left(\lambda t_{2} . t_{2}\right) k_{3} k_{4} t_{5} \\
& \operatorname{eval}_{0}\left(t \star_{2} t^{\prime}\right) k_{1} k_{2} k_{3} k_{4} t_{5}=\operatorname{eval}_{0} t k_{1}\left(\lambda f_{2} . \operatorname{eval}_{0} t^{\prime} k_{1}\left(\lambda f_{2}^{\prime} . k_{2}\left(f_{2} \circ f_{2}^{\prime}\right)\right)\right) k_{3} k_{4} t_{5} \\
& \text { eval }_{0} \varepsilon_{3} k_{1} k_{2} k_{3} k_{4} t_{5}=k_{3}\left(\lambda t_{3} . t_{3}\right) k_{4} t_{5} \\
& \operatorname{eval}_{0}\left(t \star_{3} t^{\prime}\right) k_{1} k_{2} k_{3} k_{4} t_{5}=\operatorname{eval}_{0} t k_{1} k_{2}\left(\lambda f_{3} \cdot \text { eval }_{0} t^{\prime} k_{1} k_{2}\left(\lambda f_{3}^{\prime} \cdot k_{3}\left(f_{3} \circ f_{3}^{\prime}\right)\right)\right) k_{4} t_{5} \\
& \text { eval }_{0} \varepsilon_{4} k_{1} k_{2} k_{3} k_{4} t_{5}=k_{4}\left(\lambda t_{4} \cdot t_{4}\right) t_{5} \\
& \operatorname{eval}_{0}\left(t \star_{4} t^{\prime}\right) k_{1} k_{2} k_{3} k_{4} t_{5}=\operatorname{eval}_{0} t k_{1} k_{2} k_{3}\left(\lambda f_{4} \cdot \text { eval }_{0} t^{\prime} k_{1} k_{2} k_{3}\left(\lambda f_{4}^{\prime} \cdot k_{4}\left(f_{4} \circ f_{4}^{\prime}\right)\right)\right) t_{5} \\
& \text { eval }_{0} \varepsilon_{5} k_{1} k_{2} k_{3} k_{4} t_{5}=t_{5} \\
& \operatorname{eval}_{0}\left(t \star_{5} t^{\prime}\right) k_{1} k_{2} k_{3} k_{4} t_{5}=\operatorname{eval}_{0} t k_{1} k_{2} k_{3} k_{4}\left(\text { eval }_{0} t^{\prime} k_{1} k_{2} k_{3} k_{4} t_{5}\right) \\
& \text { reify } v=v\left(\lambda f_{1} \cdot \lambda k_{2} \cdot k_{2}\left(\lambda t_{2} \cdot\left(f_{1} \varepsilon_{1}^{\mathrm{nf}}\right) \star_{2}^{\mathrm{nf}} t_{2}\right)\right) \\
& \left(\lambda f_{2} \cdot \lambda k_{3} \cdot k_{3}\left(\lambda t_{3} \cdot\left(f_{2} \varepsilon_{2}^{\mathrm{nf}}\right) \star_{3}^{\mathrm{nf}} t_{3}\right)\right) \\
& \left(\lambda f_{3} \cdot \lambda k_{4} \cdot k_{4}\left(\lambda t_{4} \cdot\left(f_{3} \varepsilon_{3}^{\mathrm{nf}}\right) \star_{4}^{\mathrm{nf}} t_{4}\right)\right) \\
& \left(\lambda f_{4} \cdot \lambda t_{5} \cdot\left(f_{4} \varepsilon_{4}^{\mathrm{nf}}\right) \star_{5}^{\mathrm{nf}} t_{5}\right) \\
& \varepsilon_{5} \\
& \text { normalize } t=\text { reify }_{0}\left(\text { eval }_{0} t\right)
\end{aligned}
$$

This normalization function uses four delimited continuations $k_{1}, k_{2}, k_{3}, k_{4}$ and five accumulators $t_{1}, t_{2}, t_{3}, t_{4}, t_{5}$ to flatten each of the successive products. In the clause of each $\varepsilon_{i}$, the continuations $k_{1}, \ldots, k_{i-1}$ are discarded, accounting for the fact that $\varepsilon_{i}$ is absorbant for $\star_{1}, \ldots, \star_{i-1}$, and the identity function is passed to $k_{i}$, accounting for the fact that $\varepsilon_{i}$ is neutral for $\star_{i}$. In the clause of each $\star_{i+1}$, the continuations $k_{1}, \ldots, k_{i}$ are duplicated and used in non-tail position, achieving the distribution of $\star_{i+1}$ over $\star_{1}, \ldots, \star_{i}$. The continuations and the accumulators are initialized in the definition of reify.

This normalization function lives at level 0 of the CPS hierarchy, but we can express it at a higher level using shift and reset. For example, uncurrying $k_{3}$ and $k_{4}$ and mapping eval 0 and reify $y_{0}$ back to direct style twice yield the following intermediate definition, which lives at level 2:

$$
\begin{aligned}
& \text { eval }_{2} x k_{1} k_{2} t_{5}=k_{1}\left(\lambda t_{1} \cdot x \star_{1}^{\mathrm{nf}} t_{1}\right) k_{2} t_{5} \\
& \text { eval }_{2} \varepsilon_{1} k_{1} k_{2} t_{5}=k_{1}\left(\lambda t_{1} \cdot t_{1}\right) k_{2} t_{5} \\
& \operatorname{eval}_{2}\left(t \star_{1} t^{\prime}\right) k_{1} k_{2} t_{5}=\operatorname{eval}_{2} t\left(\lambda f_{1} . \text { eval }_{2} t^{\prime}\left(\lambda f_{1}^{\prime} \cdot k_{1}\left(f_{1} \circ f_{1}^{\prime}\right)\right)\right) k_{2} t_{5} \\
& \text { eval }_{2} \varepsilon_{2} k_{1} k_{2} t_{5}=k_{2}\left(\lambda t_{2} . t_{2}\right) t_{5} \\
& \operatorname{eval}_{2}\left(t \star_{2} t^{\prime}\right) k_{1} k_{2} t_{5}=\text { eval }_{2} t k_{1}\left(\lambda f_{2} . \text { eval }_{2} t^{\prime} k_{1}\left(\lambda f_{2}^{\prime} . k_{2}\left(f_{2} \circ f_{2}^{\prime}\right)\right)\right) t_{5} \\
& \text { eval }_{2} \varepsilon_{3} k_{1} k_{2} t_{5}=\left(\lambda t_{3} . t_{3}, t_{5}\right) \\
& \operatorname{eval}_{2}\left(t \star_{3} t^{\prime}\right) k_{1} k_{2} t_{5}=\operatorname{let}\left(f_{3}, t_{5}\right)=\operatorname{eval}_{2} t k_{1} k_{2} t_{5} \\
& \text { in let }\left(f_{3}^{\prime}, t_{5}\right)=\text { eval }_{2} t^{\prime} k_{1} k_{2} t_{5} \\
& \text { in }\left(f_{3} \circ f_{3}^{\prime}, t_{5}\right)
\end{aligned}
$$




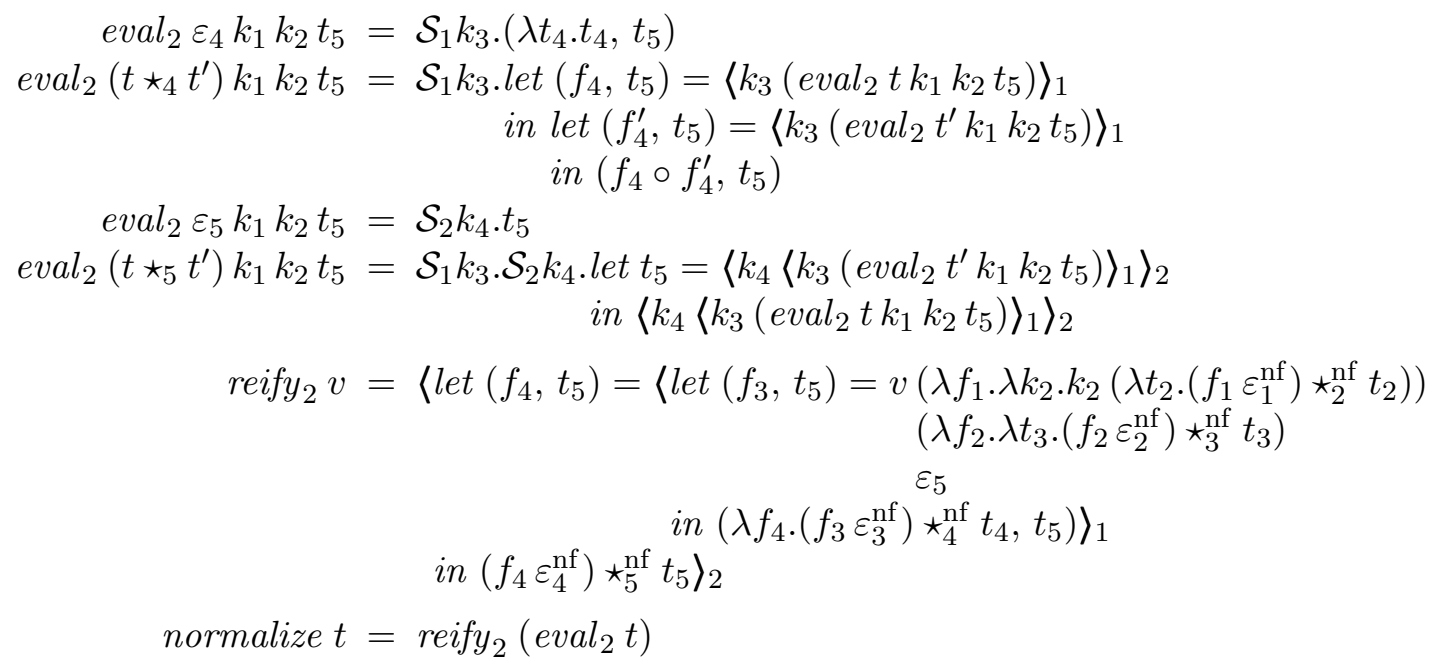

Whereas $\mathrm{eval}_{0}$ had four layered continuations, $\mathrm{eval}_{2}$ has only two layered continuations since it has been mapped back to direct style twice. Where eval 0 accesses $k_{3}$ as one of its parameters, $e^{e v a l_{2}}$ abstracts the first layer of control with shift ${ }_{1}$, and where $e v a l_{0}$ accesses $k_{4}$ as one of its parameters, eval 2 abstracts the first and the second layer of control with shift . $_{2}$

Uncurrying $k_{1}$ and $k_{2}$ and mapping eval 2 and reify $y_{2}$ back to direct style twice yield the following direct-style definition, which lives at level 4 of the CPS hierarchy:

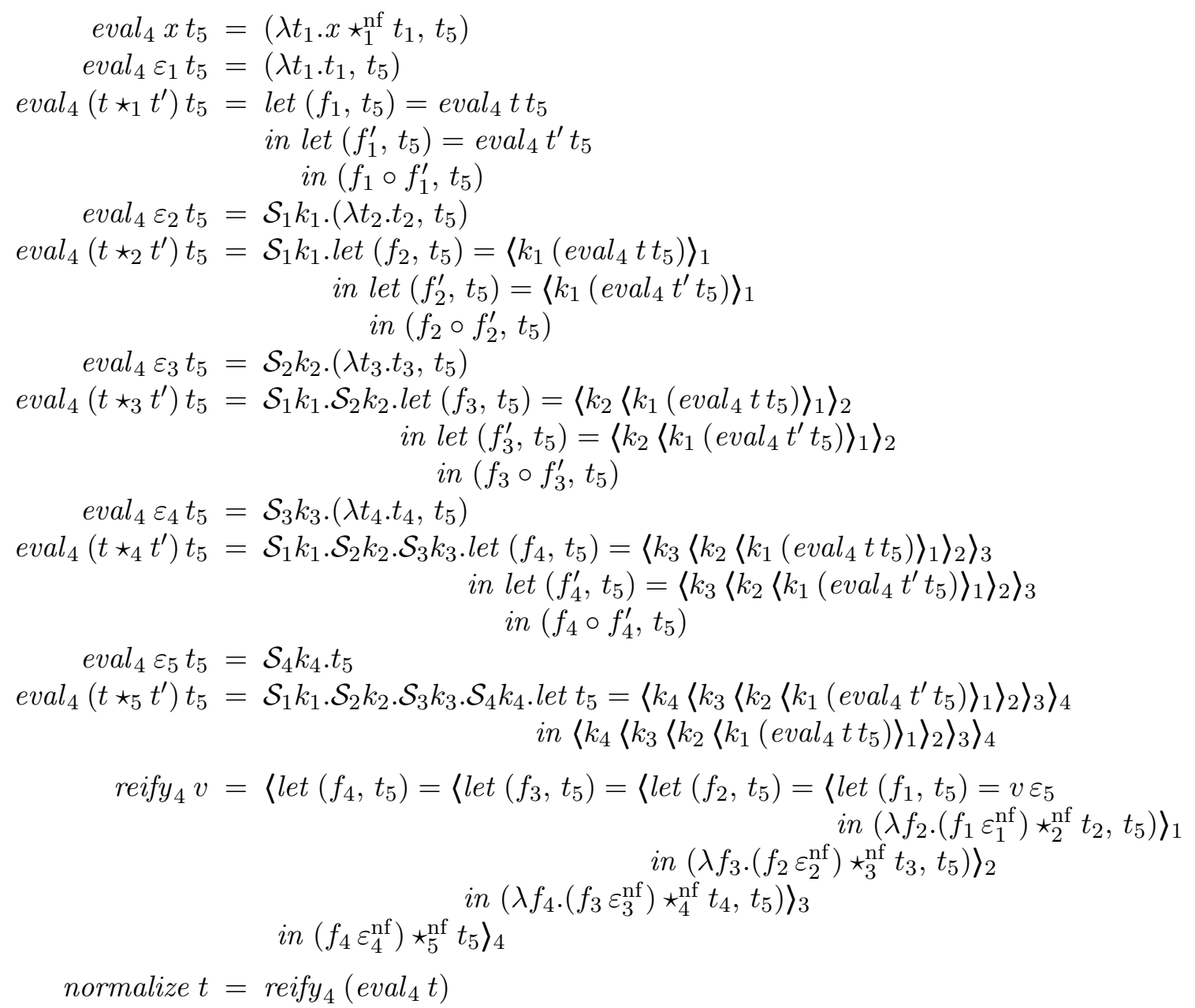


Whereas $e^{2} a l_{2}$ had two layered continuations, eval 4 has none since it has been mapped back to direct style twice. Where eval $_{2}$ accesses $k_{1}$ as one of its parameters, eval 4 abstracts the first layer of control with shift ${ }_{1}$, and where eval $_{2}$ accesses $k_{2}$ as one of its parameters, eval 4 abstracts the first and the second layer of control with shift ${ }_{2}$. Where eval $_{2}$ uses reset I $_{1}$ and

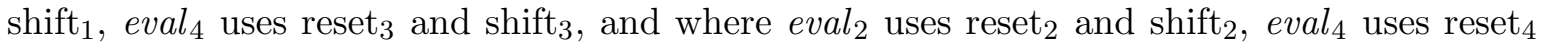
and shift $_{4}$.

\subsection{A note about efficiency}

We have implemented all the definitions of Section 6.4 as well as the intermediate versions $\mathrm{eval}_{1}$ and $\mathrm{eval}_{3}$, using Standard ML of New Jersey [31]. We have also implemented hierarchical normalization functions for other values than 5 .

For high products (i.e., in Section 6.4, for source terms using $\star_{3}$ and $\star_{4}$ ), the normalization function living at level 0 of the CPS hierarchy is the most efficient one. On the other hand, for low products (i.e., in Section 6.4, for source terms using $\star_{1}$ and $\star_{2}$ ), the normalization functions living at a higher level of the CPS hierarchy are the most efficient ones. These relative efficiencies are explained in terms of resources:

- Accessing to a continuation as an explicit parameter is more efficient than accessing to it through a control operator.

- On the other hand, the restriction of eval $_{4}$ to source terms that only use $\varepsilon_{1}$ and $\star_{1}$ is in direct style, whereas the corresponding restrictions of $e v a l_{2}$ and $e v a l_{0}$ pass a number of extra parameters. These extra parameters penalize performance.

The better performance of programs in the CPS hierarchy has already been reported for level 1 in the context of continuation-based partial evaluation [58], and it has been reported for a similar "pay as you go" reason: a program that abstracts control relatively rarely is run more efficiently in direct style with a control operator rather than in continuation-passing style.

\subsection{Summary and conclusion}

We have illustrated the CPS hierarchy with an application of normalization by evaluation that naturally involves successive layers of continuations and that demonstrates the expressive power of $\operatorname{shift}_{n}$ and reset $_{n}$.

The application also suggests alternative control operators that would fit better its continuation-based programming pattern. For example, instead of representing a delimited continuation as a function and apply it as such, we could represent it as a continuation and apply it with a throw operator as in MacLisp and Standard ML of New Jersey. For another example, instead of throwing a value to a continuation, we could specify the continuation of a computation, e.g., with a reflect ${ }_{i}$ special form. For a third example, instead of abstracting control up to a layer $n$, we could give access to each of the successive layers up to $n$, e.g., with a $\mathcal{L}_{n}$ operator. Then instead of

$$
\begin{aligned}
& \operatorname{eval}_{4}\left(t \star_{4} t^{\prime}\right) t_{5}=\mathcal{S}_{1} k_{1} \cdot \mathcal{S}_{2} k_{2} \cdot \mathcal{S}_{3} k_{3} . \text { let }\left(f_{4}, t_{5}\right)=\left\langle k_{3}\left\langle k_{2}\left\langle k_{1}\left(\text { eval }_{4} t t_{5}\right)\right\rangle_{1}\right\rangle_{2}\right\rangle_{3} \\
& \text { in let }\left(f_{4}^{\prime}, t_{5}\right)=\left\langle k_{3}\left\langle k_{2}\left\langle k_{1}\left(\text { eval }_{4} t^{\prime} t_{5}\right)\right\rangle_{1}\right\rangle_{2}\right\rangle_{3} \\
& \text { in }\left(f_{4} \circ f_{4}^{\prime}, t_{5}\right)
\end{aligned}
$$


one could write

$$
\begin{aligned}
\text { eval }_{4}\left(t \star_{4} t^{\prime}\right) t_{5}=\mathcal{L}_{3}\left(k_{1}, k_{2}, k_{3}\right) . \text { let }\left(f_{4}^{\prime}, t_{5}\right)=\text { reflect }_{3}\left(\text { eval }_{4} t t_{5}, k_{1}, k_{2}, k_{3}\right) \\
\text { in let }\left(f_{4}^{\prime}, t_{5}\right)=\text { reflect }_{3}\left(\text { eval }_{4} t^{\prime} t_{5}, k_{1}, k_{2}, k_{3}\right) \\
\text { in }\left(f_{4} \circ f_{4}^{\prime}, t_{5}\right) .
\end{aligned}
$$

Such alternative control operators can be more convenient to use, while being compatible with CPS.

\section{Conclusion and issues}

We have used CPS as a guideline to establish an operational foundation for delimited continuations. Starting from a call-by-value evaluator for $\lambda$-terms with shift and reset, we have mechanically derived the corresponding abstract machine. From this abstract machine, it is straightforward to obtain a reduction semantics of delimited control that, by construction, is compatible with CPS - both for one-step reduction and for evaluation. These results can also be established without the guideline of CPS, but less easily.

The whole approach generalizes straightforwardly to account for the $\operatorname{shift}_{n}$ and $\operatorname{reset}_{n}$ family of delimited-control operators and more generally for any control operators that are compatible with CPS. These results would be non-trivial to establish without the guideline of CPS.

Defunctionalization provides a key for connecting continuation-passing style and operational intuitions about control. Indeed most of the time, control stacks and evaluation contexts are the defunctionalized continuations of an evaluator. Defunctionalization also provides a key for identifying where operational intuitions about control go beyond CPS (see Section 4.5). We do not know whether CPS is the ultimate answer, but the present work shows yet another example of its usefulness. It is like nothing can go wrong with CPS.

Acknowledgments: We are grateful to Mads Sig Ager, Julia Lawall, Jan Midtgaard, and the referees of CW'04 and of LMCS for their comments. The third author would also like to thank Samuel Lindley for our joint initial study of the normalization functions of Section 6 .

This work is partially supported by the ESPRIT Working Group APPSEM II (http: //www.appsem.org) and by the Danish Natural Science Research Council, Grant no. 21-020474 (for the two first authors) and Grant no. 21-03-0545 (for the third author).

\section{References}

[1] Mads Sig Ager, Dariusz Biernacki, Olivier Danvy, and Jan Midtgaard. A functional correspondence between evaluators and abstract machines. In Dale Miller, editor, Proceedings of the Fifth ACM-SIGPLAN International Conference on Principles and Practice of Declarative Programming (PPDP'03), pages 8-19. ACM Press, August 2003.

[2] Mads Sig Ager, Olivier Danvy, and Jan Midtgaard. A functional correspondence between call-by-need evaluators and lazy abstract machines. Information Processing Letters, 90(5):223-232, 2004. Extended version available as the technical report BRICS-RS04-3. 
[3] Mads Sig Ager, Olivier Danvy, and Jan Midtgaard. A functional correspondence between monadic evaluators and abstract machines for languages with computational effects. Theoretical Computer Science, 2005. To appear. Extended version available as the technical report BRICS RS-04-28.

[4] Thorsten Altenkirch, Martin Hofmann, and Thomas Streicher. Categorical reconstruction of a reduction-free normalization proof. In David H. Pitt, David E. Rydeheard, and Peter Johnstone, editors, Category Theory and Computer Science, number 953 in Lecture Notes in Computer Science, pages 182-199, Cambridge, UK, August 1995. SpringerVerlag.

[5] Zena M. Ariola, Hugo Herbelin, and Amr Sabry. A type-theoretic foundation of continuations and prompts. In Kathleen Fisher, editor, Proceedings of the 2004 ACM SIGPLAN International Conference on Functional Programming, pages 40-53, Snowbird, Utah, September 2004. ACM Press.

[6] Kenichi Asai. Online partial evaluation for shift and reset. In Peter Thiemann, editor, Proceedings of the 2002 ACM SIGPLAN Workshop on Partial Evaluation and SemanticsBased Program Manipulation (PEPM 2002), SIGPLAN Notices, Vol. 37, No 3, pages 19-30, Portland, Oregon, March 2002. ACM Press.

[7] Kenichi Asai. Offline partial evaluation for shift and reset. In Nevin Heintze and Peter Sestoft, editors, Proceedings of the 2004 ACM SIGPLAN Symposium on Partial Evaluation and Semantics-Based Program Manipulation (PEPM 2004), pages 3-14, Verona, Italy, August 2003. ACM Press.

[8] Vincent Balat, Roberto Di Cosmo, and Marcelo P. Fiore. Extensional normalisation and type-directed partial evaluation for typed lambda calculus with sums. In Xavier Leroy, editor, Proceedings of the Thirty-First Annual ACM Symposium on Principles of Programming Languages, pages 64-76, Venice, Italy, January 2004. ACM Press.

[9] Vincent Balat and Olivier Danvy. Memoization in type-directed partial evaluation. In Don Batory, Charles Consel, and Walid Taha, editors, Proceedings of the 2002 ACM SIGPLAN/SIGSOFT Conference on Generative Programming and Component Engineering, number 2487 in Lecture Notes in Computer Science, pages 78-92, Pittsburgh, Pennsylvania, October 2002. Springer-Verlag.

[10] Anindya Banerjee, Nevin Heintze, and Jon G. Riecke. Design and correctness of program transformations based on control-flow analysis. In Naoki Kobayashi and Benjamin C. Pierce, editors, Theoretical Aspects of Computer Software, 4th International Symposium, TACS 2001, number 2215 in Lecture Notes in Computer Science, pages 420-447, Sendai, Japan, October 2001. Springer-Verlag.

[11] Nuel D. Belnap. How a computer should think. In Gilbert Ryle, editor, Proceedings of the Oxford International Symposium on Contemporary Aspects of Philosophy, pages 30-56, Oxford, England, 1976. Oriel Press.

[12] Ulrich Berger, Matthias Eberl, and Helmut Schwichtenberg. Normalization by evaluation. In Bernhard Möller and John V. Tucker, editors, Prospects for hardware foundations (NADA), number 1546 in Lecture Notes in Computer Science, pages 117-137, Berlin, Germany, 1998. Springer-Verlag. 
[13] Ulrich Berger and Helmut Schwichtenberg. An inverse of the evaluation functional for typed $\lambda$-calculus. In Gilles Kahn, editor, Proceedings of the Sixth Annual IEEE Symposium on Logic in Computer Science, pages 203-211, Amsterdam, The Netherlands, July 1991. IEEE Computer Society Press.

[14] Ilya Beylin and Peter Dybjer. Extracting a proof of coherence for monoidal categories from a proof of normalization for monoids. In Stefano Berardi and Mario Coppo, editors, Types for Proofs and Programs, International Workshop TYPES'95, number 1158 in Lecture Notes in Computer Science, pages 47-61, Torino, Italy, June 1995. SpringerVerlag.

[15] Małgorzata Biernacka, Dariusz Biernacki, and Olivier Danvy. An operational foundation for delimited continuations. In Thielecke [75], pages 25-33.

[16] Dariusz Biernacki and Olivier Danvy. From interpreter to logic engine by defunctionalization. In Maurice Bruynooghe, editor, Logic Based Program Synthesis and Transformation, 13th International Symposium, LOPSTR 2003, number 3018 in Lecture Notes in Computer Science, pages 143-159, Uppsala, Sweden, August 2003. Springer-Verlag.

[17] Dariusz Biernacki and Olivier Danvy. On the dynamic extent of delimited continuations. Technical Report BRICS RS-05-2, DAIMI, Department of Computer Science, University of Aarhus, Aarhus, Denmark, January 2005.

[18] Dariusz Biernacki, Olivier Danvy, and Kevin Millikin. A dynamic continuation-passing style for dynamic delimited continuations. Technical Report BRICS RS-05-5, DAIMI, Department of Computer Science, University of Aarhus, Aarhus, Denmark, February 2005.

[19] Thierry Coquand and Peter Dybjer. Intuitionistic model constructions and normalization proofs. Mathematical Structures in Computer Science, 7:75-94, 1997.

[20] Olivier Danvy. On listing list prefixes. LISP Pointers, 2(3-4):42-46, January 1989.

[21] Olivier Danvy. Type-directed partial evaluation. In Guy L. Steele Jr., editor, Proceedings of the Twenty-Third Annual ACM Symposium on Principles of Programming Languages, pages 242-257, St. Petersburg Beach, Florida, January 1996. ACM Press.

[22] Olivier Danvy. Type-directed partial evaluation. In John Hatcliff, Torben Æ. Mogensen, and Peter Thiemann, editors, Partial Evaluation - Practice and Theory; Proceedings of the 1998 DIKU Summer School, number 1706 in Lecture Notes in Computer Science, pages 367-411, Copenhagen, Denmark, July 1998. Springer-Verlag.

[23] Olivier Danvy. From reduction-based to reduction-free normalization. Technical Report BRICS RS-04-30, DAIMI, Department of Computer Science, University of Aarhus, Aarhus, Denmark, December 2004. Invited talk at the 4th International Workshop on Reduction Strategies in Rewriting and Programming (WRS 2004), Aachen, Germany, June 2, 2004. To appear in ENTCS.

[24] Olivier Danvy. On evaluation contexts, continuations, and the rest of the computation. In Thielecke [75], pages 13-23. Invited talk. 
[25] Olivier Danvy. A rational deconstruction of Landin's SECD machine. In Clemens Grelck and Frank Huch, editors, Implementation and Application of Functional Languages, 16th International Workshop, IFL'04, number 3474 in Lecture Notes in Computer Science, Lübeck, Germany, September 2004. Springer-Verlag. To appear. Extended version available as the technical report BRICS-RS-03-33.

[26] Olivier Danvy and Andrzej Filinski. A functional abstraction of typed contexts. DIKU Rapport 89/12, DIKU, Computer Science Department, University of Copenhagen, Copenhagen, Denmark, July 1989.

[27] Olivier Danvy and Andrzej Filinski. Abstracting control. In Wand [78], pages 151-160.

[28] Olivier Danvy and Andrzej Filinski. Representing control, a study of the CPS transformation. Mathematical Structures in Computer Science, 2(4):361-391, 1992.

[29] Olivier Danvy and Lasse R. Nielsen. Defunctionalization at work. In Harald Søndergaard, editor, Proceedings of the Third International ACM SIGPLAN Conference on Principles and Practice of Declarative Programming (PPDP'01), pages 162-174, Firenze, Italy, September 2001. ACM Press. Extended version available as the technical report BRICS RS-01-23.

[30] Olivier Danvy and Lasse R. Nielsen. Refocusing in reduction semantics. Technical Report BRICS RS-04-26, DAIMI, Department of Computer Science, University of Aarhus, Aarhus, Denmark, November 2004. A preliminary version appears in the informal proceedings of the Second International Workshop on Rule-Based Programming (RULE 2001), Electronic Notes in Theoretical Computer Science, Vol. 59.4.

[31] Olivier Danvy and Zhe Yang. An operational investigation of the CPS hierarchy. In S. Doaitse Swierstra, editor, Proceedings of the Eighth European Symposium on Programming, number 1576 in Lecture Notes in Computer Science, pages 224-242, Amsterdam, The Netherlands, March 1999. Springer-Verlag.

[32] Scott Draves. Implementing bit-addressing with specialization. In Mads Tofte, editor, Proceedings of the 1997 ACM SIGPLAN International Conference on Functional Programming, pages 239-250, Amsterdam, The Netherlands, June 1997. ACM Press.

[33] Peter Dybjer and Andrzej Filinski. Normalization and partial evaluation. In Gilles Barthe, Peter Dybjer, Luís Pinto, and João Saraiva, editors, Applied Semantics - Advanced Lectures, number 2395 in Lecture Notes in Computer Science, pages 137-192, Caminha, Portugal, September 2000. Springer-Verlag.

[34] R. Kent Dybvig, Simon Peyton-Jones, and Amr Sabry. A monadic framework for subcontinuations. Available at http://www.cs.indiana.edu/〜sabry/research.html, February 2005.

[35] Matthias Felleisen. The Calculi of $\lambda$-v-CS Conversion: A Syntactic Theory of Control and State in Imperative Higher-Order Programming Languages. PhD thesis, Department of Computer Science, Indiana University, Bloomington, Indiana, August 1987.

[36] Matthias Felleisen. The theory and practice of first-class prompts. In Jeanne Ferrante and Peter Mager, editors, Proceedings of the Fifteenth Annual ACM Symposium on Principles 
of Programming Languages, pages 180-190, San Diego, California, January 1988. ACM Press.

[37] Matthias Felleisen and Matthew Flatt. Programming languages and lambda calculi. Unpublished lecture notes. http://www.ccs.neu.edu/home/matthias/3810-w02/ readings.html, 1989-2003.

[38] Matthias Felleisen and Daniel P. Friedman. Control operators, the SECD machine, and the $\lambda$-calculus. In Martin Wirsing, editor, Formal Description of Programming Concepts III, pages 193-217. Elsevier Science Publishers B.V. (North-Holland), Amsterdam, 1986.

[39] Matthias Felleisen, Daniel P. Friedman, Bruce Duba, and John Merrill. Beyond continuations. Technical Report 216, Computer Science Department, Indiana University, Bloomington, Indiana, February 1987.

[40] Matthias Felleisen, Mitchell Wand, Daniel P. Friedman, and Bruce F. Duba. Abstract continuations: A mathematical semantics for handling full functional jumps. In Robert (Corky) Cartwright, editor, Proceedings of the 1988 ACM Conference on Lisp and Functional Programming, pages 52-62, Snowbird, Utah, July 1988. ACM Press.

[41] Andrzej Filinski. Representing monads. In Hans-J. Boehm, editor, Proceedings of the Twenty-First Annual ACM Symposium on Principles of Programming Languages, pages 446-457, Portland, Oregon, January 1994. ACM Press.

[42] Andrzej Filinski. Representing layered monads. In Alex Aiken, editor, Proceedings of the Twenty-Sixth Annual ACM Symposium on Principles of Programming Languages, pages 175-188, San Antonio, Texas, January 1999. ACM Press.

[43] Andrzej Filinski. Normalization by evaluation for the computational lambda-calculus. In Samson Abramsky, editor, Typed Lambda Calculi and Applications, 5th International Conference, TLCA 2001, number 2044 in Lecture Notes in Computer Science, pages 151-165, Kraków, Poland, May 2001. Springer-Verlag.

[44] Martin Gasbichler and Michael Sperber. Final shift for call/cc: direct implementation of shift and reset. In Simon Peyton Jones, editor, Proceedings of the 2002 ACM SIGPLAN International Conference on Functional Programming, SIGPLAN Notices, Vol. 37, No. 9, pages 271-282, Pittsburgh, Pennsylvania, September 2002. ACM Press.

[45] Matthew L. Ginsberg. Multivalued logics: a uniform approach to reasoning in artificial intelligence. Computational Intelligence, 4:265-316, 1988.

[46] Bernd Grobauer and Zhe Yang. The second Futamura projection for type-directed partial evaluation. Higher-Order and Symbolic Computation, 14(2/3):173-219, 2001.

[47] Carl Gunter, Didier Rémy, and Jon G. Riecke. A generalization of exceptions and control in ML-like languages. In Simon Peyton Jones, editor, Proceedings of the Seventh ACM Conference on Functional Programming and Computer Architecture, pages 12-23, La Jolla, California, June 1995. ACM Press.

[48] Thérèse Hardin, Luc Maranget, and Bruno Pagano. Functional runtime systems within the lambda-sigma calculus. Journal of Functional Programming, 8(2):131-172, 1998. 
[49] Simon Helsen and Peter Thiemann. Two flavors of offline partial evaluation. In Jieh Hsiang and Atsushi Ohori, editors, Advances in Computing Science - ASIAN'98, number 1538 in Lecture Notes in Computer Science, pages 188-205, Manila, The Philippines, December 1998. Springer-Verlag.

[50] Robert Hieb and R. Kent Dybvig. Continuations and concurrency. In Proceedings of the Second ACM SIGPLAN Symposium on Principles 83 Practice of Parallel Programming, SIGPLAN Notices, Vol. 25, No. 3, pages 128-136, Seattle, Washington, March 1990. ACM Press.

[51] Robert Hieb, R. Kent Dybvig, and Claude W. Anderson, III. Subcontinuations. Lisp and Symbolic Computation, 5(4):295-326, December 1993.

[52] John Hughes. A novel representation of lists and its application to the function "reverse". Information Processing Letters, 22(3):141-144, 1986.

[53] Yukiyoshi Kameyama. Axioms for delimited continuations in the CPS hierarchy. In Jerzy Marcinkowski and Andrzej Tarlecki, editors, Computer Science Logic, 18th International Workshop, CSL 2004, 13th Annual Conference of the EACSL, Proceedings, volume 3210 of Lecture Notes in Computer Science, pages 442-457, Karpacz, Poland, September 2004. Springer.

[54] Yukiyoshi Kameyama and Masahito Hasegawa. A sound and complete axiomatization of delimited continuations. In Olin Shivers, editor, Proceedings of the 2003 ACM SIGPLAN International Conference on Functional Programming, pages 177-188, Uppsala, Sweden, August 2003. ACM Press.

[55] Richard Kelsey, William Clinger, and Jonathan Rees, editors. Revised ${ }^{5}$ report on the algorithmic language Scheme. Higher-Order and Symbolic Computation, 11(1):7-105, 1998.

[56] Yoshiki Kinoshita. A bicategorical analysis of E-categories. Mathematica Japonica, 47(1):157-169, 1998.

[57] Peter J. Landin. The mechanical evaluation of expressions. The Computer Journal, 6(4):308-320, 1964.

[58] Julia L. Lawall and Olivier Danvy. Continuation-based partial evaluation. In Carolyn L. Talcott, editor, Proceedings of the 1994 ACM Conference on Lisp and Functional Programming, LISP Pointers, Vol. VII, No. 3, pages 227-238, Orlando, Florida, June 1994. ACM Press.

[59] Per Martin-Löf. About models for intuitionistic type theories and the notion of definitional equality. In Proceedings of the Third Scandinavian Logic Symposium (1972), volume 82 of Studies in Logic and the Foundation of Mathematics, pages 81-109. NorthHolland, 1975.

[60] Eugenio Moggi. Notions of computation and monads. Information and Computation, 93:55-92, 1991. 
[61] Luc Moreau and Christian Queinnec. Partial continuations as the difference of continuations, a duumvirate of control operators. In Manuel Hermenegildo and Jaan Penjam, editors, Sixth International Symposium on Programming Language Implementation and Logic Programming, number 844 in Lecture Notes in Computer Science, pages 182-197, Madrid, Spain, September 1994. Springer-Verlag.

[62] Chethan R. Murthy. Control operators, hierarchies, and pseudo-classical type systems: A-translation at work. In Olivier Danvy and Carolyn L. Talcott, editors, Proceedings of the First ACM SIGPLAN Workshop on Continuations (CW 1992), Technical report STAN-CS-92-1426, Stanford University, pages 49-72, San Francisco, California, June 1992.

[63] Lasse R. Nielsen. A denotational investigation of defunctionalization. Technical Report BRICS RS-00-47, DAIMI, Department of Computer Science, University of Aarhus, Aarhus, Denmark, December 2000.

[64] Gordon D. Plotkin. Call-by-name, call-by-value and the $\lambda$-calculus. Theoretical Computer Science, 1:125-159, 1975.

[65] Gordon D. Plotkin. A structural approach to operational semantics. Technical Report FN-19, DAIMI, Department of Computer Science, University of Aarhus, Aarhus, Denmark, September 1981.

[66] Christian Queinnec and Bernard Serpette. A dynamic extent control operator for partial continuations. In Robert (Corky) Cartwright, editor, Proceedings of the Eighteenth Annual ACM Symposium on Principles of Programming Languages, pages 174-184, Orlando, Florida, January 1991. ACM Press.

[67] John C. Reynolds. Definitional interpreters for higher-order programming languages. Higher-Order and Symbolic Computation, 11(4):363-397, 1998. Reprinted from the proceedings of the 25th ACM National Conference (1972), with a foreword.

[68] Chung-chieh Shan. Shift to control. In Olin Shivers and Oscar Waddell, editors, Proceedings of the 2004 ACM SIGPLAN Workshop on Scheme and Functional Programming, Snowbird, Utah, September 2004.

[69] Dorai Sitaram. Models of Control and their Implications for Programming Language Design. PhD thesis, Computer Science Department, Rice University, Houston, Texas, April 1994.

[70] Dorai Sitaram and Matthias Felleisen. Control delimiters and their hierarchies. Lisp and Symbolic Computation, 3(1):67-99, January 1990.

[71] Dorai Sitaram and Matthias Felleisen. Reasoning with continuations II: Full abstraction for models of control. In Wand [78], pages 161-175.

[72] Guy L. Steele Jr. Rabbit: A compiler for Scheme. Master's thesis, Artificial Intelligence Laboratory, Massachusetts Institute of Technology, Cambridge, Massachusetts, May 1978. Technical report AI-TR-474. 
[73] Eijiro Sumii. An implementation of transparent migration on standard Scheme. In Matthias Felleisen, editor, Proceedings of the Workshop on Scheme and Functional Programming, pages 61-64, Montréal, Canada, September 2000. Rice Technical Report 00-368.

[74] Eijiro Sumii and Naoki Kobayashi. A hybrid approach to online and offline partial evaluation. Higher-Order and Symbolic Computation, 14(2/3):101-142, 2001.

[75] Hayo Thielecke, editor. Proceedings of the Fourth ACM SIGPLAN Workshop on Continuations, Technical report CSR-04-1, Department of Computer Science, Queen Mary's College, Venice, Italy, January 2004.

[76] Peter Thiemann. Combinators for program generation. Journal of Functional Programming, 9(5):483-525, 1999.

[77] Philip Wadler. Monads and composable continuations. LISP and Symbolic Computation, 7(1):39-55, January 1994.

[78] Mitchell Wand, editor. Proceedings of the 1990 ACM Conference on Lisp and Functional Programming, Nice, France, June 1990. ACM Press.

[79] Yong Xiao, Amr Sabry, and Zena M. Ariola. From syntactic theories to interpreters: Automating proofs of unique decomposition. Higher-Order and Symbolic Computation, 14(4):387-409, 2001. 


\section{Recent BRICS Report Series Publications}

RS-05-11 Małgorzata Biernacka, Dariusz Biernacki, and Olivier Danvy. An Operational Foundation for Delimited Continuations in the CPS Hierarchy. March 2005. iii+42 pp. Appears in Thielecke, editor, 4th ACM SIGPLAN Workshop on Continuations, CW'04 Proceedings, Association for Computing Machinery (ACM) SIGPLAN Technical Reports CSR-04-1, 2004, pages 25-33. This version supersedes BRICS RS-04-29.

RS-05-10 Dariusz Biernacki and Olivier Danvy. A Simple Proof of a Folklore Theorem about Delimited Control. March 2005. ii+11 pp.

RS-05-9 Gudmund Skovbjerg Frandsen and Peter Bro Miltersen. Reviewing Bounds on the Circuit Size of the Hardest Functions. March 2005. 6 pp. To appear in Information Processing Letters.

RS-05-8 Peter D. Mosses. Exploiting Labels in Structural Operational Semantics. February 2005. 15 pp. Appears in Fundamenta Informaticae, 60:17-31, 2004.

RS-05-7 Peter D. Mosses. Modular Structural Operational Semantics. February 2005. 46 pp. Appears in Journal of Logic and Algebraic Programming, 60-61:195-228, 2004.

RS-05-6 Karl Krukow and Andrew Twigg. Distributed Approximation of Fixed-Points in Trust Structures. February 2005. 41 pp.

RS-05-5 A Dynamic Continuation-Passing Style for Dynamic Delimited Continuations. Dariusz Biernacki and Olivier Danvy and Kevin Millikin. February 2005.

RS-05-4 Andrzej Filinski and Henning Korsholm Rohde. Denotational Aspects of Untyped Normalization by Evaluation. February 2005.

RS-05-3 Olivier Danvy and Mayer Goldberg. There and Back Again. January 2005. iii+16 pp. Extended version of an article to appear in Fundamenta Informatica. This version supersedes BRICS RS-02-12.

RS-05-2 Dariusz Biernacki and Olivier Danvy. On the Dynamic Extent of Delimited Continuations. January 2005. ii+30 pp.

RS-05-1 Mayer Goldberg. On the Recursive Enumerability of FixedPoint Combinators. January 2005. 7 pp. Superseedes BRICS report RS-04-25. 